\title{
A POSTERIORI ERROR ESTIMATES FOR FULLY DISCRETE SCHEMES FOR THE TIME DEPENDENT STOKES PROBLEM
}

\author{
E. BÄNSCH, F. KARAKATSANI, AND C. G. MAKRIDAKIS
}

\begin{abstract}
This work is devoted to a posteriori error analysis of fully discrete finite element approximations to the time dependent Stokes system. The space discretization is based on popular stable spaces, including Crouzeix-Raviart and Taylor-Hood finite element methods. Implicit Euler is applied for the time discretization. The finite element spaces are allowed to change with time steps and the projection steps include alternatives that is hoped to cope with possible numerical artifices and the loss of the discrete incompressibility of the schemes. The final estimates are of optimal order in $L^{\infty}\left(L^{2}\right)$ for the velocity error.
\end{abstract}

\section{INTRODUCTION}

This work is devoted to a posteriori error analysis of fully discrete finite element approximations to the time dependent Stokes system. The bounds derived herein can be seen as a necessary step towards the error control of the nonlinear Navier Stokes equations. Although between reach, this goal involves other nontrivial steps as well, which are currently under investigation. A posteriori estimates, in addition of their use as a mathematical tool to back up computational methods, can be used in the design of adaptive algorithms. Such algorithms can detect the areas of interest of the solution in order to auto adjust the numerical method during the computation. In recent years, adaptive methods for the computation of solutions of partial differential equations (PDEs) are quite popular. In fact, such methods utilising self adjusted meshes have important benefits approximating PDEs with solutions that exhibit nontrivial characteristics.

This paper is part of our ongoing effort to understand and to address open problems related to error control of time dependent problems related in one way or another to Stokes evolutions, see e.g., $[6,7,26,25]$. In what follows, we consider the time dependent Stokes equations; we derive and analyse a posteriori error estimators for full discretizations allowing changing in time finite element spaces. Our estimators allow for special projections during the mesh evolution process which may restore the discrete divergence free status of the approximations when mesh change with time is present.

To be more precise, the following problem is considered: Let $\Omega$ be a bounded connected domain of $\mathbb{R}^{d}(d=2)$ with sufficiently smooth boundary for our purposes and $T$ be a positive constant. We consider the time-dependent Stokes problem describing the flow of a viscous

1991 Mathematics Subject Classification. 65M15, 65M50, 65N15.

Key words and phrases. A posteriori error estimators, time dependent Stokes, reconstruction, adaptivity, mesh change, Crouzeix-Raviart element.

The third author was partially supported by the ITN Network "Modelling and Computations for Schocks and Interfaces" funded by H2020-EU.1.3.1-Project 642768. 
incompressible fluid:

$$
\begin{aligned}
\mathbf{u}_{t}-\Delta \mathbf{u}+\nabla p=\mathbf{f} & \text { in } \Omega \times] 0, T[ \\
\operatorname{div} \mathbf{u}=0 & \text { in } \Omega \times] 0, T[ \\
\mathbf{u}=0 & \text { on } \partial \Omega \times] 0, T[ \\
\mathbf{u}(\cdot, 0)=\mathbf{u}_{0}(\cdot) & \text { in } \Omega .
\end{aligned}
$$

Here, the unknowns are the velocity $\mathbf{u}$ and the pressure $p$; the external force $\mathbf{f}$ and the initial velocity $\mathbf{u}_{0}$ are data of our problem.

A posteriori estimates and associated adaptive algorithms for time dependent problems is a challenging area, both for theory and computations. A key issue, often underestimated, is the theoretical and computational problem associated with the need of spatial mesh modification with time. Spatial mesh movement is a necessity for evolution phenomena, however its influence to both the algorithm behavior and to the error estimates is somewhat unexpected. As first noticed in the classical article by Dupont [19], too careless mesh modification may lead to convergence to wrong solutions. Subtle phenomena in the a posteriori analysis of parabolic problems were noticed and analysed in $[6,7]$.

A related problem, maybe even harder, concerns evolutions with elliptic constraints as, for instance, the solenoidal condition for incompressible fluids. Changing the mesh (usually) results in a change of the discrete constraint, which in turn may have a devastating effect at least on the Lagrangian multiplier (i.e. the pressure for incompressible fluids), if the transfer of the primal variable from one step to the next one is realized by just standard operators (interpolation or $L^{2}$ projection), see $[9,12,13]$.

Roughly speaking, the main structure of an algorithm which permits mesh redistribution with time has the form: Given the approximation $u_{n}$ at the time step $n$, which belongs to a finite dimensional space $V_{n}$ (reflecting the space discretization method)

1a : choose the next space $V_{n+1}$,

1b : project $u_{n}$ to the new space $V_{n+1}$ to get $\tilde{u}_{n}$,

1c: use $\tilde{u}_{n}$ as starting value to perform the evolution step in $V_{n+1}$ to obtain the new approximation $u_{n+1} \in V_{n+1}$.

Standard schemes involve only step (1c) (uniform or nonuniform mesh). The presence of (1a) and (1b) are in most of the cases neglected in the analysis, but as noted earlier (1a) and (1b) have fundamental influence on the qualitative behavior of the schemes. Such influence becomes evident in pressure pollution in Navier-Stokes solvers. In fact, mesh redistribution can pollute in a severe way the pressure approximation, see Fig 1. There, a computational example based on van Karman vortex shedding highlighting this effect is presented. Computational results are shown both with a fixed mesh (right) and for meshes that are refined randomly (left). It is evident that in the second case the pressure heavily oscillates at the refinement areas. For this calculation the software package NAVIER [5] with P2/P1 Taylor-Hood elements was used. This numerical artifact is probably due to the fact that the new projected approximations are non discrete divergence free in the new space. This is the motivation for introducing a certain projection in Definition 2.2 onto the space of discrete divergence free velocities on the new mesh, when modifying the spatial discretization spaces as time progresses.

Our estimators are based on the methodology developed in $[29,28]$ for space discrete and fully discrete and in $[1,2]$ for time discrete schemes. The analysis includes rather general pairs of finite elements including for instance the celebrated nonconforming Crouzeix-Raviart elements and the conforming Taylor-Hood elements. Moreover, the results can be extended to finite volume discretizations by adopting arguments of [15] to treat the "elliptic" estimators in Section 5. 
Alternative estimators for various discretization methods for problem at hand, mainly based on the direct comparison of $u$ and the piecewise linear interpolant in time of the numerical solution $U$, can be found in, e.g., $[11,10,34]$. The time discretization methods are Implicit Euler, or more general $\theta$ methods and the finite element spaces can vary. In these works a norm which includes among other terms the error in $L^{2}\left([0, T] ; H^{1}(\Omega)\right.$ of the velocity is considered. Lower bounds are provided for most of the estimators involved. In [34] the final bounds involve additional terms which account for the lack of divergence free of the approximations. Our approach differs essentially on the methodological choice to work with reconstructions in space and time. This choice permits us to avoid restrictions on the choice of norms. On the other hand lower bounds are more involved and are related to discrete inf-sup conditions. This discussion is postponed for a forthcoming work.
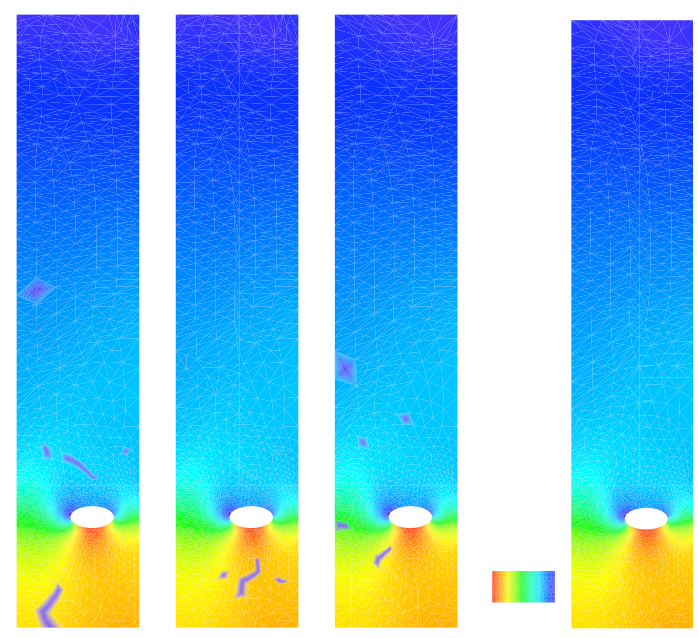

Figure 1. A computational example highlighting the effect of the mesh modification on the pressure. The experiment is based on the solution of the full Navier-Stokes equations, in a special geometry, referred as von Karman vortex shedding. Left: the pressure approximation oscillates at the areas of refinement. Right: the pressure approximations without mesh change.

The rest of this paper is organized as follows. In the next section we introduce the necessary notation, the numerical scheme and our assumptions on the finite element spaces. In Section 3 we define the space-time reconstructions and we describe the structure of the elliptic type a posteriori estimators which two successive spaces have to fulfill. In Section 4 we present the error analysis estimating the errors of the velocity and the pressure. In Section 5 we verify the hypotheses regarding the elliptic estimators, and hence we show that our results are applicable when we use a) the Crouzeix-Raviart spaces and b) the Taylor-Hood elements. Finally, in Section 6 we present numerical experiments indicating that our estimators are of optimal order and that the mesh change effects can be controlled by the estimator.

\section{Preliminaries}

In this section we introduce the necessary notation for our analysis and the fully discrete scheme. 
2.1. The variational formulation. Let $\mathbf{H}:=\left(L^{2}(\Omega)\right)^{d}$ be the usual Lebesque space equipped with the inner product

$$
\langle\mathbf{f}, \mathbf{g}\rangle=\int_{\Omega} \mathbf{f}(x) \cdot \mathbf{g}(x) d x
$$

and $\mathbf{V}:=\left(H_{0}^{1}(\Omega)\right)^{d}, S:=\left\{\phi \in L^{2}(\Omega): \int_{\Omega} \phi(x) d x=0\right\}$, and let $\mathbf{V}^{\star}:=\left(H^{-1}(\Omega)\right)^{d}$ be the dual of $\mathbf{V}$. Denote the norms on $\mathbf{H}, S, \mathbf{V}$ and $\mathbf{V}^{*}$ by $\|\cdot\|_{\mathbf{H}},\|\cdot\|_{S},\|\cdot\|_{\mathbf{V}}$ and $\|\cdot\|_{\mathbf{V}^{*}}$, respectively. More generally, for $k \in \mathbb{N}$ and $D \subseteq \mathbb{R}^{d}$ denote by $\|\cdot\|_{k, D}$ the norm in $H^{k}(D)$ and by $|\cdot|_{k, D}$ the corresponding semi-norm. If $D=\Omega$, the subscript $D$ is dropped. Likewise, if $k=0$ the subscript $k$ is omitted.

Let $a(\cdot, \cdot)$ and $b(\cdot, \cdot)$ be the bilinear forms defined as

$$
a(\mathbf{u}, \mathbf{v})=\int_{\Omega} \nabla \mathbf{u}: \nabla \mathbf{v} d x, \quad \mathbf{u}, \mathbf{v} \in \mathbf{V}
$$

and

$$
b(\mathbf{u}, q)=-\int_{\Omega}(\operatorname{div} \mathbf{u}) q d x, \quad \mathbf{u} \in \mathbf{V}, q \in S .
$$

We assume that $\mathbf{f} \in L^{2}\left(0, T ; \mathbf{V}^{\star}\right)$ and $\mathbf{u}_{0} \in \mathbf{H}$, so that (1.1) admits a unique weak solution $(\mathbf{u}, p) \in L^{\infty}(0, T ; \mathbf{H}) \cap L^{2}(0, T ; \mathbf{V}) \times L^{\infty}(0, T ; S)$ satisfying

$$
u(\cdot, 0)=\mathbf{u}_{0}, \quad \text { a.e. in } \Omega
$$

and, for a.e. $t \in] 0, T[$,

$$
\begin{aligned}
\left\langle\mathbf{u}_{t}(t), \mathbf{v}\right\rangle+a(\mathbf{u}(t), \mathbf{v})+b(\mathbf{v}, p(t)) & =\langle\mathbf{f}(t), \mathbf{v}\rangle \quad \forall \mathbf{v} \in \mathbf{V}, \\
b(\mathbf{u}(t), q) & =0 \quad \forall q \in S .
\end{aligned}
$$

In the sequel we will assume that the data of the problem have sufficient (additional) regularity for our results to hold. For detailed smoothness results and corresponding regularity requirements on the data of $(2.4)$ see $[32,18]$. In particular we assume that the stationary Stokes problem is $H^{2} \times H^{1}$-regular, see also Section 5 .

In the sequel the "divergence-free" subspaces of $\mathbf{V}$ and $\mathbf{H}$ will be useful. Following, e.g. [18], let $\mathscr{V}=\{\mathbf{v} \in \mathscr{D}(\Omega)$, div $\mathbf{v}=0\}$, where $\mathscr{D}(\Omega)=C_{0}^{\infty}(\Omega)$. We then define $\mathbf{J}$ and $\mathbf{Z}$ to be the closures of $\mathscr{V}$ in $\mathbf{H}$ and $\mathbf{V}$, respectively. The following characterization holds, [18] Section 1.4.,

$$
\mathbf{J}=\{\mathbf{v} \in \mathbf{H}: \operatorname{div} \mathbf{v}=0 \text { and } \mathbf{v} \cdot \mathbf{n}=0\}, \quad \mathbf{Z}=\{\mathbf{v} \in \mathbf{V}: \operatorname{div} \mathbf{v}=0\} .
$$

Then, naturally, problem (2.4) is reduced to the following two problems: find $\mathbf{u} \in \mathbf{Z}$ such that

$$
\left\langle\mathbf{u}_{t}(t), \mathbf{v}\right\rangle+a(\mathbf{u}(t), \mathbf{v})=\langle\mathbf{f}(t), \mathbf{v}\rangle \quad \forall \mathbf{v} \in \mathbf{Z},
$$

and $p \in S$ such that

$$
b(\mathbf{v}, p(t))=-\left\langle\mathbf{u}_{t}(t), \mathbf{v}\right\rangle-a(\mathbf{u}(t), \mathbf{v})+\langle\mathbf{f}(t), \mathbf{v}\rangle \quad \forall \mathbf{v} \in \mathbf{V} .
$$

Well-posedness of problem (2.6) follows from the coercivity of the bilinear form $a(\cdot, \cdot)$, namely

$$
\|\mathbf{v}\|_{\mathbf{V}}^{2} \leq a(\mathbf{v}, \mathbf{v}) \quad \forall \mathbf{v} \in \mathbf{V}
$$

and the well-posedness of problem (2.7) follows from the continuous inf-sup condition

$$
\beta\|p\| \leq \sup _{\mathbf{w} \in \mathbf{V}} \frac{b(\mathbf{w}, p)}{\|\mathbf{w}\|_{\mathbf{V}}} \quad \forall p \in S,
$$

where $\beta>0$. 
Definition 2.1 (The Stokes Operator). Let $P: \mathbf{H} \rightarrow \mathbf{J}$ be the $L^{2}$-projection of $\mathbf{H}$ onto $\mathbf{J}$ and $\tilde{\Delta}: \mathbf{H}^{2} \cap \mathbf{Z} \subset \mathbf{J} \rightarrow \mathbf{J}$ be the Stokes operator, [22, 23], namely the $L^{2}$-projection of the Laplace operator onto $\mathbf{J}$,

$$
\langle\tilde{\Delta} \mathbf{v}, \mathbf{w}\rangle=\langle\Delta \mathbf{v}, \mathbf{w}\rangle \quad \forall \mathbf{w} \in \mathbf{J}
$$

2.2. Discretization. Let $0=t^{0}<t^{1}<\cdots<t^{N}=T$ be a partition of $] 0, T$ [ into subintervals $I_{n}:=\left(t^{n-1}, t^{n}\right]$, and denote by $k_{n}:=t^{n}-t^{n-1}$ the time steps.

For convenience the shorthand notations $u^{m}:=u\left(t^{m}\right)$ and $f^{m}:=f\left(t^{m}\right)$ are used throughout the rest of the paper.

Let $\mathcal{M}$ be a macro-triangulation of $\Omega$. We associate with each time $t^{n}, 0 \leq n \leq N$, a refinement $\mathcal{T}_{n}$ of $\mathcal{M}$ into disjoint $d$-simplices $K$. We assume that all triangulations $\left(\mathcal{T}_{n}\right)_{n \in\{0,1, \ldots, N\}}$ are derived from $\mathcal{M}$ by using an admissible refinement procedure, e.g., the bisection-based refinement procedure used in ALBERTA-FEM toolbox, cf. $[4,30]$. Let $h_{n}$ denote the local mesh-size function of $\mathcal{T}_{n}$ defined by

$$
h_{n}(x):=\operatorname{diam}(K), \quad K \in \mathcal{T}_{n} \text { and } x \in K .
$$

We also assume that the aspect ratios of all the elements are uniformly bounded with respect to $n, n=0, \ldots, N$, and the intersection of two different elements is either empty, or consists of a common vertex, a common edge, or a common face.

For a $d$-simplex $K$ we denote by $\mathcal{E}(K)$ the set of sides of $K$ (edges in $d=2$ or faces in $d=3$ ) and by $\Sigma(K) \subset \mathcal{E}(K)$ the set of sides of $K$ which do not belong to the boundary $\partial \Omega$. Furthermore, for each triangulation $\mathcal{T}_{n}, 0 \leq n \leq N$, we introduce the sets $\mathcal{E}_{n}:=\cup_{K \in \mathcal{T}_{n}} \mathcal{E}(K)$ and $\Sigma_{n}:=\cup_{K \in \mathcal{T}_{n}} \Sigma(K)$.

Let $\mathcal{T}, \mathcal{T}^{\prime}$ denote two refinements of $\mathcal{M}$. We write $\mathcal{T} \leq \mathcal{T}^{\prime}$ if $\mathcal{T}^{\prime}$ is a refinement of $\mathcal{T}$. Given two successive triangulations $\mathcal{T}_{n-1}$ and $\mathcal{T}_{n}$, we introduce their finest common coarsening $\hat{\mathcal{T}}_{n}$, that is the finest triangulation satisfying $\hat{\mathcal{T}}_{n} \leq \mathcal{T}_{n-1}$ and $\hat{\mathcal{T}}_{n} \leq \mathcal{T}_{n}$, and the coarsest common refinement $\check{\mathcal{T}}_{n}$, that is the coarsest triangulation that satisfies $\mathcal{T}_{n-1} \leq \check{\mathcal{T}}_{n}$ and $\mathcal{T}_{n} \leq \check{\mathcal{T}}_{n}$. Let $\hat{h}_{n}:=\max \left(h_{n}, h_{n-1}\right)$, and $\check{\Sigma}_{n}$ and $\hat{\Sigma}_{n}$ be the sets of the interior sides corresponding to $\check{\mathcal{T}}_{n}$ and $\hat{\mathcal{T}}_{n}$, respectively, namely $\check{\Sigma}_{n}:=\cup_{K \in \check{\mathcal{T}}_{n}} \Sigma(K)$ and $\hat{\Sigma}_{n}:=\cup_{K \in \hat{\mathcal{T}}_{n}} \Sigma(K)$.

2.3. Finite element spaces. With each triangulation $\mathcal{T}_{n}$ a pair of finite element spaces $\left(\mathbf{V}_{h}^{n}, S_{h}^{n}\right)$ (i.e. spaces consisting of functions that are piecewise smooth) is associated which is "appropriate" for the discretization of the Stokes problem.

Our analysis is valid for diverse conforming (i.e. $\mathbf{V}_{h}^{n} \subset \mathbf{V}$ ) as well as nonconforming finite element spaces (i.e. $\mathbf{V}_{h}^{n} \not \subset \mathbf{V}$ ). In the case of nonconforming elements it is assumed that $a(\cdot, \cdot)$ can be extended to $\left(\mathbf{V}_{h}^{n}+\mathbf{V}\right) \times\left(\mathbf{V}_{h}^{n}+\mathbf{V}\right)$, and $b(\cdot, \cdot)$ can be extended to $\left(\mathbf{V}_{h}^{n}+\mathbf{V}\right) \times S$. In addition, for $\mathbf{v} \in \mathbf{V}_{h}^{n}$, with a slight abuse of notation, we still denote its norm in $\mathbf{V}_{h}^{n}$ by $\|v\|_{\mathbf{V}}$ (understood in the elementwise sense).

Furthermore, we require the finite element spaces to fulfill the following assumption.

Assumption 1. The broken semi-norm

$$
\left\|\mathbf{v}_{n}\right\|_{\mathbf{V}}^{2}=a\left(\mathbf{v}_{n}, \mathbf{v}_{n}\right) \quad \forall \mathbf{v}_{n} \in \mathbf{V}_{h}^{n}
$$

is in fact a norm on $\mathbf{V}_{h}^{n}$ and there exists a constant $\beta_{n}>0$ such that

$$
\beta_{n}=\inf _{q_{n} \in S_{h}^{n}} \sup _{\mathbf{v}_{n} \in \mathbf{V}_{h}^{n}} \frac{b\left(\mathbf{v}_{n}, q_{n}\right)}{\left\|\mathbf{v}_{n}\right\| \mathbf{v}\left\|q_{n}\right\|} .
$$


Note that Assumption 1 is fulfilled for instance for the classical Taylor-Hood element as well as for the Crouzeix-Raviart element of lowest order [16, 32].

Moreover, we introduce the "discrete divergence-free" subspace $\mathbf{Z}_{h}^{n}$ of $\mathbf{V}_{h}^{n}$, that is

$$
\mathbf{Z}_{h}^{n}:=\left\{\boldsymbol{\varphi}_{n} \in \mathbf{V}_{h}^{n}: b\left(\boldsymbol{\varphi}, q_{n}\right)=0 \quad \forall q_{n} \in S_{h}^{n}\right\} .
$$

Let $\mathbf{n}_{e}$ denote a unit normal vector to $e \in \Sigma_{n}$ with fixed but arbitrary orientation and $x \in e$. We denote by $\llbracket v \rrbracket_{e}$ and $\llbracket \mathbf{v} \rrbracket_{e}$ the jump of a possibly discontinuous scalar or vector valued function, respectively, across $e$ in the direction of $\mathbf{n}_{e}$, that is

$$
\begin{aligned}
& \llbracket v \rrbracket_{e}(x):=\lim _{\delta \rightarrow 0}\left[v\left(x+\delta \mathbf{n}_{e}\right)-v\left(x-\delta \mathbf{n}_{e}\right)\right], \\
& \llbracket \mathbf{v} \rrbracket_{e}(x):=\lim _{\delta \rightarrow 0}\left[\mathbf{v}\left(x+\delta \mathbf{n}_{e}\right)-\mathbf{v}\left(x-\delta \mathbf{n}_{e}\right)\right] \cdot \mathbf{n}_{e} .
\end{aligned}
$$

In addition, we shall use the following notation for functions $v$ defined in a piecewise sense

$$
\begin{aligned}
\left\|h_{n}^{i} v\right\|_{\mathcal{T}_{n}} & =\left(\sum_{K \in \mathcal{T}_{n}}\left\|h_{K}^{i} v\right\|_{K}^{2}\right)^{1 / 2} \\
\text { and } \quad\left\|h_{n}^{i+\frac{1}{2}} \llbracket \nabla \rrbracket v\right\|_{\Sigma_{n}} & =\left(\sum_{e \in \Sigma_{n}}\left\|h_{e}^{i+\frac{1}{2}} \llbracket \nabla v \rrbracket_{e}\right\|_{e}^{2}\right)^{1 / 2}, \quad i=1,2 .
\end{aligned}
$$

2.4. Discrete operators. Let $P_{0}^{n}: \mathbf{H} \rightarrow \mathbf{V}_{h}^{n}$ be the $L^{2}$-projection of $\mathbf{H}$ onto $\mathbf{V}_{h}^{n}$ and $\tilde{P}_{0}^{n}: \mathbf{H} \rightarrow$ $\mathbf{Z}_{h}^{n}$ be the $L^{2}$-projection of $\mathbf{H}$ onto $\mathbf{Z}_{h}^{n}$ and $\Delta_{h}^{n}: \mathbf{V}_{h}^{n} \rightarrow \mathbf{V}_{h}^{n}$ be the discrete Laplace operator onto $\mathbf{V}_{h}^{n}$,

$$
\left\langle-\Delta_{h}^{n} \mathbf{w}_{n}, \boldsymbol{\varphi}_{n}\right\rangle=a\left(\mathbf{w}_{n}, \boldsymbol{\varphi}_{n}\right) \quad \forall \boldsymbol{\varphi}_{n} \in \mathbf{V}_{h}^{n} .
$$

In addition, let $\tilde{\Delta}_{h}^{n}: \mathbf{Z}_{h}^{n} \rightarrow \mathbf{Z}_{h}^{n}$ be the discrete Stokes operator, [22, 23],

$$
\left\langle-\tilde{\Delta}_{h}^{n} \mathbf{w}_{n}, \boldsymbol{\varphi}_{n}\right\rangle=a\left(\mathbf{w}_{n}, \boldsymbol{\varphi}_{n}\right) \quad \forall \boldsymbol{\varphi}_{n} \in \mathbf{Z}_{h}^{n},
$$

and $B_{h}^{n}: S_{h}^{n} \rightarrow \mathbf{V}_{h}^{n}$,

$$
\left\langle B_{h}^{n} q_{n}, \boldsymbol{\varphi}_{n}\right\rangle=b\left(\boldsymbol{\varphi}_{n}, q_{n}\right) \quad \forall \boldsymbol{\varphi}_{n} \in \mathbf{V}_{h}^{n}
$$

In principle, our a posteriori error analysis is independent of the transfer of $\mathbf{U}^{n-1} \in \mathbf{V}_{h}^{n-1}$ to $\mathbf{V}_{h}^{n}$. The error introduced by this transfer operator will be accounted for by the error estimators anyway. However, due to the high sensitivity of the pressure approximation to mesh modification, see for instance $[9,12,13]$, in order to avoid severe pressure oscillations, it is highly recommended to project the approximations on a given time level to the discretely divergence free space of the next time. To this end we introduce the following projection.

Definition 2.2. For each $\mathbf{v}_{n-1} \in \mathbf{V}_{h}^{n-1}$, we define the pair $\left(\Pi^{n} \mathbf{v}_{n-1}, q\right) \in \mathbf{Z}_{h}^{n} \times S_{h}^{n}$ as the solution of the stationary Stokes problem

$$
\left\{\begin{aligned}
\bar{a}\left(\Pi^{n} \mathbf{v}_{n-1}, \boldsymbol{\varphi}_{n}\right)+b\left(\boldsymbol{\varphi}_{n}, q\right) & =\bar{a}\left(\mathbf{v}_{n-1}, \boldsymbol{\varphi}_{n}\right) & & \forall \boldsymbol{\varphi}_{n} \in \mathbf{V}_{h}^{n}, \\
b\left(\Pi^{n} \mathbf{v}_{n-1}, \phi_{n}\right) & =0 & & \forall \phi_{n} \in S_{h}^{n}
\end{aligned}\right.
$$

where

$$
\bar{a}\left(\mathbf{v}_{n-1}, \boldsymbol{\varphi}_{n}\right):=\lambda\left\langle\mathbf{v}_{n-1}, \boldsymbol{\varphi}_{n}\right\rangle+a\left(\mathbf{v}_{n-1}, \boldsymbol{\varphi}_{n}\right), \quad \lambda \geq 0 .
$$

Notice that since $\bar{a}\left(\mathbf{v}_{n-1}, \boldsymbol{\varphi}_{n}\right)$ is a regular perturbation of $a\left(\mathbf{v}_{n-1}, \boldsymbol{\varphi}_{n}\right)$ which is still positive and symmetric, this operator is well defined. Given $\mathbf{v}_{n-1}$, the computation of $\Pi^{n} \mathbf{v}_{n-1}$ requires the solution of a modified stationary Stokes problem. Notice that our estimators below, are still valid when we use alternative definitions for $\Pi^{n} \mathbf{v}_{n-1}$. 
2.5. Fully discrete scheme. A backward Euler-Galerkin scheme is used to discretize problem (2.4): find $\left(\mathbf{U}^{n}, P^{n}\right) \in \mathbf{V}_{h}^{n} \times S_{h}^{n}, 1 \leq n \leq N$, such that $\mathbf{U}^{0}$ is a suitable approximation to $\mathbf{u}_{0}$ and that, for all $n, 1 \leq n \leq N$, and for all $\left(\boldsymbol{\varphi}_{n}, q_{n}\right) \in \mathbf{V}_{h}^{n} \times S_{h}^{n}$,

$$
\left\{\begin{array}{rlrl}
\left\langle\frac{\mathbf{U}^{n}-\Pi^{n} \mathbf{U}^{n-1}}{k_{n}}, \boldsymbol{\varphi}_{n}\right\rangle+a\left(\mathbf{U}^{n}, \boldsymbol{\varphi}_{n}\right)+b\left(\boldsymbol{\varphi}_{n}, P^{n}\right) & =\left\langle\mathbf{f}^{n}, \boldsymbol{\varphi}_{n}\right\rangle & & \forall \boldsymbol{\varphi}_{n} \in \mathbf{V}_{h}^{n}, \\
b\left(\mathbf{U}^{n}, q_{n}\right)=0 & & \forall q_{n} \in S_{h}^{n},
\end{array}\right.
$$

where $\Pi^{n}$ is the projection defined in Eq. (2.20).

By using Definition (2.14), Eq. (2.22) is equivalent to the following formulation: find $\mathbf{U}^{n} \in \mathbf{Z}_{h}^{n}$ such that

$$
\left\langle\frac{\mathbf{U}^{n}-\Pi^{n} \mathbf{U}^{n-1}}{k_{n}}, \boldsymbol{\varphi}_{n}\right\rangle+a\left(\mathbf{U}^{n}, \boldsymbol{\varphi}_{n}\right)=\left\langle\mathbf{f}^{n}, \boldsymbol{\varphi}_{n}\right\rangle \quad \forall \boldsymbol{\varphi}_{n} \in \mathbf{Z}_{h}^{n}
$$

and then determine $P^{n} \in S_{h}^{n}$ such that

$$
b\left(\boldsymbol{\varphi}_{n}, P^{n}\right)=-\left\langle\frac{\mathbf{U}^{n}-\Pi^{n} \mathbf{U}^{n-1}}{k_{n}}, \boldsymbol{\varphi}_{n}\right\rangle-a\left(\mathbf{U}^{n}, \boldsymbol{\varphi}_{n}\right)+\left\langle\mathbf{f}^{n}, \boldsymbol{\varphi}_{n}\right\rangle \quad \forall \boldsymbol{\varphi}_{n} \in \mathbf{V}_{h}^{n}
$$

For each $h_{n}$ and $1 \leq n \leq N$, the existence and the uniqueness of the $\left(\mathbf{U}^{n}, P^{n}\right)$ follows from Assumption 1, see for instance [14, p. 248] and [20, p. 59].

Remark 2.1. Note that a priori analysis requires $\beta_{n}$ to be bounded from below independent of $h_{n}$, in order to get optimal error estimates, [20, p. 117]. In contrast, a posteriori analysis is based on the stability of the continuous problem and therefore (formally) does not need this assumption, see for instance [8] for convergence results without inf-sup condition. However, this does not mean that we advocate using elements not satisfying an inf-sup condition.

2.6. The fully discrete scheme in compact form. According to (2.17) and (2.19) the fully discrete scheme may also be written in the following operator form:

$$
k_{n}^{-1}\left(\mathbf{U}^{n}-\Pi^{n} \mathbf{U}^{n-1}\right)-\Delta_{h}^{n} \mathbf{U}^{n}+B_{h}^{n} P^{n}=P_{0}^{n} \mathbf{f}^{n} .
$$

Indeed, in view of (2.22), we have

$$
\begin{aligned}
\left\langle k_{n}^{-1}\left(\mathbf{U}^{n}-\Pi^{n} \mathbf{U}^{n-1}\right)-\Delta_{h}^{n} \mathbf{U}^{n}+\right. & \left.B_{h}^{n} P^{n}-P_{0}^{n} \mathbf{f}^{n}, \boldsymbol{\varphi}_{n}\right\rangle=\left\langle k_{n}^{-1}\left(\mathbf{U}^{n}-\Pi^{n} \mathbf{U}^{n-1}\right), \boldsymbol{\varphi}_{n}\right\rangle \\
& +a\left(\mathbf{U}^{n}, \boldsymbol{\varphi}_{n}\right)+b\left(\boldsymbol{\varphi}_{n}, P^{n}\right)-\left\langle\mathbf{f}^{n}, \boldsymbol{\varphi}_{n}\right)=0 \quad \forall \boldsymbol{\varphi}_{n} \in \mathbf{V}_{h}^{n} .
\end{aligned}
$$

Since $k_{n}^{-1}\left(\mathbf{U}^{n}-\Pi^{n} \mathbf{U}^{n-1}\right)-\Delta_{h}^{n} \mathbf{U}^{n}+B_{h}^{n} P^{n}-P_{0}^{n} \mathbf{f}^{n} \in \mathbf{V}_{h}^{n}$, we can conclude the desired result.

\section{Space-time Reconstructions}

3.1. Stokes reconstruction. The Stokes reconstruction, introduced in [27], is a key point in the a posteriori error analysis. One of its main advantages in the error analysis is that this reconstruction restores the divergence free condition of the error quantity, which otherwise is lost, and thus allows the application of various analytical techniques to derive the estimates. We recall its definition and basic facts.

Definition 3.1 (Stokes reconstruction). For a given pair $\left(\mathbf{w}_{n}, q_{n}\right) \in \mathbf{V}_{h}^{n} \times S_{h}^{n}$ we define its Stokes reconstruction $\mathcal{R}^{n}\left(\mathbf{w}_{n}, q_{n}\right)=\left(\mathcal{R}_{u}^{n}\left(\mathbf{w}_{n}, q_{n}\right), \mathcal{R}_{p}^{n}\left(\mathbf{w}_{n}, q_{n}\right)\right) \in \mathbf{V} \times S$ as the solution of the stationary Stokes problem

$$
\left\{\begin{aligned}
a\left(\mathcal{R}_{u}^{n}\left(\mathbf{w}_{n}, q_{n}\right), \boldsymbol{\varphi}\right)+b\left(\boldsymbol{\varphi}, \mathcal{R}_{p}^{n}\left(\mathbf{w}_{n}, q_{n}\right)\right) & =\left\langle-\Delta_{h}^{n} \mathbf{w}_{n}+B_{h}^{n} q_{n}, \boldsymbol{\varphi}\right\rangle \quad \forall \boldsymbol{\varphi} \in \mathbf{V}, \\
b\left(\mathcal{R}_{u}^{n}\left(\mathbf{w}_{n}, q_{n}\right), q\right) & =0 \quad \forall q \in S
\end{aligned}\right.
$$


A crucial property of the Stokes reconstruction $\mathcal{R}^{n}\left(\mathbf{w}_{n}, q_{n}\right)$ is expressed by the following lemma.

Lemma 3.1. Let $\mathcal{R}^{n}\left(U^{n}, P^{n}\right) \in \mathbf{V} \times S$ be the unique solution of problem (3.1) and $\left(\mathbf{W}^{n}, Q^{n}\right) \in$ $\mathbf{V}_{h}^{n} \times S_{h}^{n}$ be its finite element solution, that is:

$$
\left\{\begin{aligned}
a\left(\mathbf{W}^{n}, \boldsymbol{\varphi}_{n}\right)+b\left(\boldsymbol{\varphi}_{n}, Q^{n}\right) & =\left\langle-\Delta_{h}^{n} \mathbf{U}^{n}+B_{h}^{n} P^{n}, \boldsymbol{\varphi}_{n}\right\rangle \quad \forall \boldsymbol{\varphi}_{n} \in \mathbf{V}_{h}^{n}, \\
b\left(\mathbf{W}^{n}, q_{n}\right) & =0 \quad \forall q_{n} \in S_{h}^{n}
\end{aligned}\right.
$$

Then $\mathbf{W}^{n}=\mathbf{U}^{n}$ and $Q^{n}=P^{n}$.

Proof. Let $\mathbf{v}_{n} \in \mathbf{Z}_{h}^{n}$; then

$$
\left\langle B_{h}^{n} P^{n}, \mathbf{v}_{n}\right\rangle=b\left(\mathbf{v}_{n}, P^{n}\right)=0
$$

and

$$
a\left(\mathbf{W}^{n}, \mathbf{v}_{n}\right)=\left\langle-\Delta_{h}^{n} \mathbf{U}^{n}+B_{h}^{n} P^{n}, \mathbf{v}_{n}\right\rangle=-\left\langle\tilde{\Delta}_{h}^{n} \mathbf{U}^{n}, \mathbf{v}_{n}\right\rangle=a\left(\mathbf{U}^{n}, \mathbf{v}_{n}\right) .
$$

Now

$$
a\left(\mathbf{U}^{n}-\mathbf{W}^{n}, \mathbf{v}_{n}\right)=0 \quad \forall \mathbf{v}_{n} \in \mathbf{Z}_{h}^{n},
$$

and therefore, since $\mathbf{U}^{n}, \mathbf{W}^{n} \in \mathbf{Z}_{h}^{n}$, we get $\mathbf{U}^{n}=\mathbf{W}^{n}$. Further, since for all $\boldsymbol{\varphi}_{n} \in \mathbf{V}_{h}^{n}$,

$$
a\left(\mathbf{W}^{n}-\mathbf{U}^{n}, \boldsymbol{\varphi}_{n}\right)=0
$$

we get

$$
b\left(\boldsymbol{\varphi}_{n}, P^{n}-Q^{n}\right)=0 \quad \forall \boldsymbol{\varphi}_{n} \in \mathbf{V}_{h}^{n} .
$$

Due to the discrete inf-sup assumption (2.13) it holds $Q^{n}=P^{n}$. Thus, $\left(\mathbf{U}^{n}, P^{n}\right) \in \mathbf{Z}_{h}^{n} \times$ $S_{h}^{n}$ is the finite element solution of the stationary Stokes equations whose exact solution is $\mathcal{R}^{n}\left(\mathbf{U}^{n}, P^{n}\right)$.

Remark 3.2 (Alternative definition of the Stokes reconstruction). The Stokes reconstruction $\mathcal{R}^{n}\left(\mathbf{U}^{n}, P^{n}\right)$ of $\left(\mathbf{U}^{n}, P^{n}\right)$ may equivalently be defined as follows

$$
\left\{\begin{array}{c}
a\left(\mathcal{R}_{u}^{n}\left(\mathbf{U}^{n}, P^{n}\right), \boldsymbol{\varphi}\right)+b\left(\boldsymbol{\varphi}, \mathcal{R}_{p}^{n}\left(\mathbf{U}^{n}, P^{n}\right)\right)=\left\langle\mathbf{f}^{n}-\frac{\mathbf{U}^{n}-\Pi^{n} \mathbf{U}^{n-1}}{k_{n}}, \boldsymbol{\varphi}\right\rangle \quad \forall \boldsymbol{\varphi} \in \mathbf{V}, \\
b\left(\mathcal{R}_{u}^{n}\left(\mathbf{U}^{n}, P^{n}\right), q\right)=0 \quad \forall q \in S .
\end{array}\right.
$$

3.2. Interpolation in time. Define the piecewise linear in time function $(\mathbf{U}, P):] 0, T\left[\rightarrow \mathbf{Z}_{h}^{n} \times\right.$ $S_{h}^{n}$ by linearly interpolating between the nodal values $\left(\mathbf{U}^{n-1}, P^{n-1}\right)$ and $\left(\mathbf{U}^{n}, P^{n}\right)$,

$$
(\mathbf{U}(t), P(t)):=\ell_{0}^{n}(t)\left(\mathbf{U}^{n-1}, P^{n-1}\right)+\ell_{1}^{n}(t)\left(\mathbf{U}^{n}, P^{n}\right), \quad t \in I_{n},
$$

and the piecewise linear function $(\hat{\mathbf{U}}, \hat{P}):] 0, T[\rightarrow \mathbf{Z} \times S$ by linearly interpolating between the nodal values $\mathcal{R}^{n-1}\left(\mathbf{U}^{n-1}, P^{n-1}\right)$ and $\mathcal{R}^{n}\left(\mathbf{U}^{n}, P^{n}\right)$

$$
(\hat{\mathbf{U}}(t), \hat{P}(t)):=\ell_{0}^{n}(t) \mathcal{R}^{n-1}\left(\mathbf{U}^{n-1}, P^{n-1}\right)+\ell_{1}^{n}(t) \mathcal{R}^{n}\left(\mathbf{U}^{n}, P^{n}\right) \quad t \in I_{n},
$$

where

$$
\ell_{0}^{n}(t)=\frac{t^{n}-t}{k_{n}} \quad \text { and } \quad \ell_{1}^{n}(t)=\frac{t-t^{n-1}}{k_{n}}, \quad t \in I_{n} .
$$

In addition, we introduce the following notation

$$
\partial \mathbf{v}^{n}:=\frac{\mathbf{v}^{n}-\mathbf{v}^{n-1}}{k_{n}} \quad \text { and } \quad \bar{\partial} \mathbf{v}^{n}:=\frac{\mathbf{v}^{n}-\Pi^{n} \mathbf{v}^{n-1}}{k_{n}}
$$


3.3. Assumptions on elliptic estimators. Our analysis is based on the availability of a posteriori error estimators for finite element approximations of the stationary Stokes problem: find $(\mathbf{w}, q) \in \mathbf{V} \times S$ such that

$$
\begin{aligned}
a(\mathbf{w}, \boldsymbol{\varphi})+b(\mathbf{v}, q) & =\langle\mathbf{g}, \boldsymbol{\varphi}\rangle \quad \forall \boldsymbol{\varphi} \in \mathbf{V}, \\
b(\mathbf{w}, \phi) & =0 \quad \forall \phi \in S,
\end{aligned}
$$

where $\mathbf{g} \in \mathbf{V}^{\star}$ is given. Let $\left(\mathbf{w}_{n}, q_{n}\right) \in \mathbf{V}_{h}^{n} \times S_{h}^{n}$ be the corresponding finite element solution, that is

$$
\begin{aligned}
a\left(\mathbf{w}_{n}, \boldsymbol{\varphi}_{n}\right)+b\left(\boldsymbol{\varphi}_{n}, q_{n}\right) & =\left\langle\mathbf{g}, \boldsymbol{\varphi}_{n}\right\rangle \quad \forall \boldsymbol{\varphi}_{n} \in \mathbf{V}_{h}^{n} \\
b\left(\mathbf{w}_{n}, \phi_{n}\right) & =0 \quad \forall \phi_{n} \in S_{h}^{n} .
\end{aligned}
$$

In what follows, a general assumption is made on the availability of a posteriori estimators for the stationary problem. For concrete pairs of finite element spaces, the estimator has to be worked out. For most of the standard finite element spaces for the Stokes problem, these estimators are available; for Crouzeix-Raviart and Taylor-Hood elements see Section 5 for explicit formulas. In view of Lemma 3.1 the assumption can be formulated with the help of the Stokes reconstruction.

Assumption 2. Let $\left(\mathbf{w}_{n-1}, q_{n-1}\right) \in \mathbf{Z}_{h}^{n-1} \times S_{h}^{n-1}$ and $\left(\mathbf{w}_{n}, q_{n}\right) \in \mathbf{Z}_{h}^{n} \times S_{h}^{n}$ be given. We assume the existence of a posteriori error estimator functions $\eta_{u}=\eta_{u}\left(\mathbf{w}_{n}, q_{n}\right)$ and $\eta_{u, \partial}=$ $\eta_{u, \partial}\left(\partial\left(\mathbf{w}_{n}, q_{n}\right)\right)$ fulfilling

$$
\left\|\left(I-\mathcal{R}_{u}^{n}\right)\left(\mathbf{w}_{n}, q_{n}\right)\right\| \leq \eta_{u}\left(\mathbf{w}_{n}, q_{n}\right)
$$

and

$$
\left\|\partial\left(I-\mathcal{R}_{u}^{n}\right)\left(\mathbf{w}_{n}, q_{n}\right)\right\| \leq \eta_{u, \partial}\left(\partial\left(\mathbf{w}_{n}, q_{n}\right)\right) .
$$

Notice that the second estimator will involve both spaces $\mathbf{Z}_{h}^{n-1} \times S_{h}^{n-1}$ and $\mathbf{Z}_{h}^{n} \times S_{h}^{n}$. Further, there exists an estimator for the pressure, $\eta_{p}=\eta_{p}\left(\mathbf{w}_{n}, q_{n}\right)$ such that

$$
\left\|\left(I-\mathcal{R}_{p}^{n}\right)\left(\mathbf{w}_{n}, q_{n}\right)\right\| \leq \eta_{p}\left(\mathbf{w}_{n}, q_{n}\right) .
$$

Note that the above assumption requires a velocity estimator for the difference of finite element functions on $\mathcal{T}_{n-1}$ and $\mathcal{T}_{n}$, respectively, i.e. including mesh changes.

For the rest of this article we assume that Assumptions 1, 2 are fulfilled. In Section 5 we verify Assumption 2 and we compute the corresponding estimator functions for the Crouzeix-Raviart and Taylor-Hood finite element spaces.

\section{ERror AnALYsis}

4.1. Error equation. Split the total error $\mathbf{e}:=\mathbf{U}-\mathbf{u}$ for the velocity as

$$
\mathbf{e}=\hat{\mathbf{e}}-\boldsymbol{\sigma},
$$

where

$$
\boldsymbol{\sigma}:=\hat{\mathbf{U}}-\mathbf{U}, \quad \hat{\mathbf{e}}:=\hat{\mathbf{U}}-\mathbf{u} .
$$

The error $\boldsymbol{\sigma}$ is an elliptic reconstruction error and may be bounded by the elliptic error estimator as follows:

Lemma 4.1 (Elliptic estimator). For $m=1, \ldots, N$, the following estimate holds

$$
\max _{0 \leq t \leq t^{m}}\|\boldsymbol{\sigma}(t)\| \leq \mathcal{E}_{m}^{\text {ell }} \quad \text { with } \quad \mathcal{E}_{m}^{\text {ell }}:=\max _{0 \leq n \leq m} \eta_{u}\left(\mathbf{U}^{n}, P^{n}\right)
$$

with $\eta_{u}$ from Assumption 2. 
Proof. Since

$$
\boldsymbol{\sigma}\left(t^{n}\right)=\left(\mathcal{R}_{u}^{n}-I\right)\left(\mathbf{U}^{n}, P^{n}\right)
$$

set $\mathbf{w}_{n}=\mathbf{U}^{n}, q_{n}=P^{n}$ in (3.14). Then $\boldsymbol{\sigma}$ is bounded by

$$
\left\|\boldsymbol{\sigma}\left(t^{n}\right)\right\| \leq \eta_{u}\left(\left(\mathbf{U}^{n}, P^{n}\right)\right) .
$$

The rest is immediate.

Let $\hat{\varepsilon}$ be defined by $\hat{\varepsilon}(t):=\mathcal{R}_{p}^{n}\left(\mathbf{U}^{n}, P^{n}\right)-p(t)$ for $t \in I_{n}$. Then the error $(\hat{\mathbf{e}}, \hat{\varepsilon})$ satisfies the following modified time-dependent Stokes equations. This error equation is fundamental in the course of proving a posteriori estimates.

Lemma 4.2 (Error equation). For each $\varphi \in \mathbf{V}$ it holds

$$
\begin{aligned}
\left\langle\hat{\mathbf{e}}_{t}(t), \boldsymbol{\varphi}\right\rangle+a(\hat{\mathbf{e}}(t), \boldsymbol{\varphi}) & +b(\boldsymbol{\varphi}, \hat{\varepsilon}(t))=\left\langle\boldsymbol{\sigma}_{t}(t), \boldsymbol{\varphi}(t)\right\rangle+a\left(\hat{\mathbf{U}}(t)-\mathcal{R}_{u}^{n}\left(\mathbf{U}^{n}, P^{n}\right), \boldsymbol{\varphi}\right) \\
& +k_{n}^{-1}\left\langle\Pi^{n} \mathbf{U}^{n-1}-\mathbf{U}^{n-1}, \boldsymbol{\varphi}\right\rangle+\left\langle P_{0}^{n} \mathbf{f}^{n}-\mathbf{f}(t), \boldsymbol{\varphi}\right\rangle, \quad t \in I_{n} .
\end{aligned}
$$

Proof. In view of the Stokes reconstruction (3.1) and the operator form (2.25) of the discrete system one obtains

$$
a\left(\mathcal{R}_{u}^{n}\left(\mathbf{U}^{n}, P^{n}\right), \boldsymbol{\varphi}\right)+b\left(\boldsymbol{\varphi}, \mathcal{R}_{p}^{n}\left(\mathbf{U}^{n}, P^{n}\right)\right)=-k_{n}^{-1}\left\langle\mathbf{U}^{n}-\Pi^{n} \mathbf{U}^{n-1}, \boldsymbol{\varphi}\right\rangle+\left\langle P_{0}^{n} \mathbf{f}^{n}, \boldsymbol{\varphi}\right\rangle
$$

for each $n=1, \ldots, N$. Moreover,

$$
\begin{aligned}
\left\langle\hat{\mathbf{U}}_{t}, \boldsymbol{\varphi}\right)+a(\hat{\mathbf{U}}, \boldsymbol{\varphi})+b\left(\boldsymbol{\varphi}, \mathcal{R}_{p}^{n}\left(\mathbf{U}^{n}, P^{n}\right)\right)= & k_{n}^{-1}\left\langle\mathcal{R}_{u}^{n}\left(\mathbf{U}^{n}, P^{n}\right)-\mathcal{R}_{u}^{n-1}\left(\mathbf{U}^{n-1}, P^{n-1}\right), \boldsymbol{\varphi}\right\rangle \\
& +a\left(\hat{\mathbf{U}}-\mathcal{R}_{u}^{n}\left(\mathbf{U}^{n}, P^{n}\right), \boldsymbol{\varphi}\right) \\
& +a\left(\mathcal{R}_{u}^{n}\left(\mathbf{U}^{n}, P^{n}\right), \boldsymbol{\varphi}\right)+b\left(\boldsymbol{\varphi}, \mathcal{R}_{p}^{n}\left(\mathbf{U}^{n}, P^{n}\right)\right),
\end{aligned}
$$

from which, according to (4.3), we obtain

$$
\begin{aligned}
\left\langle\hat{\mathbf{U}}_{t}, \boldsymbol{\varphi}\right)+a(\hat{\mathbf{U}}, \boldsymbol{\varphi})+ & b\left(\boldsymbol{\varphi}, \mathcal{R}_{p}^{n}\left(\mathbf{U}^{n}, P^{n}\right)\right)=k_{n}^{-1}\left\langle\mathcal{R}_{u}^{n}\left(\mathbf{U}^{n}, P^{n}\right)-\mathcal{R}_{u}^{n-1}\left(\mathbf{U}^{n-1}, P^{n-1}\right), \boldsymbol{\varphi}\right\rangle \\
& +a\left(\hat{\mathbf{U}}-\mathcal{R}_{u}^{n}\left(\mathbf{U}^{n}, P^{n}\right), \boldsymbol{\varphi}\right)-k_{n}^{-1}\left\langle\mathbf{U}^{n}-\Pi^{n} \mathbf{U}^{n-1}, \boldsymbol{\varphi}\right\rangle+\left\langle P_{0}^{n} \mathbf{f}^{n}, \boldsymbol{\varphi}\right\rangle,
\end{aligned}
$$

i.e.

$$
\begin{aligned}
\left\langle\hat{\mathbf{U}}_{t}, \boldsymbol{\varphi}\right\rangle+a(\hat{\mathbf{U}}, \boldsymbol{\varphi})+ & b\left(\boldsymbol{\varphi}, \mathcal{R}_{p}^{n}\left(\mathbf{U}^{n}, P^{n}\right)\right)=k_{n}^{-1}\left\langle\left(\mathcal{R}_{u}^{n}\left(\mathbf{U}^{n}, P^{n}\right)-\mathcal{R}_{u}^{n-1}\left(\mathbf{U}^{n-1}, P^{n-1}\right)\right)-\left(\mathbf{U}^{n}-\mathbf{U}^{n-1}\right), \boldsymbol{\varphi}\right\rangle \\
& +a\left(\hat{\mathbf{U}}-\mathcal{R}_{u}^{n}\left(\mathbf{U}^{n}, P^{n}\right), \boldsymbol{\varphi}\right)+k_{n}^{-1}\left\langle\Pi^{n} \mathbf{U}^{n-1}-\mathbf{U}^{n-1}, \boldsymbol{\varphi}\right\rangle+\left\langle P_{0}^{n} \mathbf{f}^{n}, \boldsymbol{\varphi}\right\rangle .
\end{aligned}
$$

In view of (3.8) and (3.9) we have

$$
\begin{aligned}
\left\langle\hat{\mathbf{U}}_{t}, \boldsymbol{\varphi}\right\rangle+a(\hat{\mathbf{U}}, \boldsymbol{\varphi})+ & b\left(\boldsymbol{\varphi}, \mathcal{R}_{p}^{n}\left(\mathbf{U}^{n}, P^{n}\right)\right)=\left\langle(\hat{\mathbf{U}}-\mathbf{U})_{t}, \boldsymbol{\varphi}\right\rangle \\
& +a\left(\hat{\mathbf{U}}-\mathcal{R}_{u}^{n}\left(\mathbf{U}^{n}, P^{n}\right), \boldsymbol{\varphi}\right)+k_{n}^{-1}\left\langle\Pi^{n} \mathbf{U}^{n-1}-\mathbf{U}^{n-1}, \boldsymbol{\varphi}\right\rangle+\left\langle P_{0}^{n} \mathbf{f}^{n}, \boldsymbol{\varphi}\right\rangle .
\end{aligned}
$$

Subtracting Eq. (2.4) from the above equation yields Eq. (4.2).

We are now in a state to formulate and prove a posteriori estimates. First, an estimate (Lemma 4.3) for the velocity error $\hat{\mathbf{e}}(t)$ is readily derived. From this, with the help of a sequence of lemmas, concrete estimators for the velocity in $L^{\infty}\left(L^{2}\right)$ are given in Theorem 4.1. In the second subsection, in Theorem 4.2, an estimate for the pressure error is presented. 


\subsection{A posteriori estimates for the velocity.}

Lemma 4.3 (A posteriori estimate for $\hat{\mathbf{e}}$ ). The following estimate is valid

$$
\max _{t \in\left[0, t^{m}\right]}\left\{\|\hat{\mathbf{e}}(t)\|^{2}+\int_{0}^{t}\|\nabla \hat{\mathbf{e}}(s)\|^{2} d s\right\} \leq\|\hat{\mathbf{e}}(0)\|^{2}+\mathcal{J}_{m}^{\mathrm{T}}+\mathcal{J}_{m}^{\mathrm{S}}+\mathcal{J}_{m}^{\mathrm{D}}+\mathcal{J}_{m}^{\mathrm{C}},
$$

where

$\mathcal{J}_{m}^{\mathrm{T}}:=\sum_{n=1}^{m} \int_{t^{n-1}}^{t^{n}}\left|a\left(\hat{\mathbf{U}}(t)-\mathcal{R}_{u}^{n}\left(\mathbf{U}^{n}, P^{n}\right), \hat{\mathbf{e}}(t)\right)\right| d t, \quad \mathcal{J}_{m}^{\mathrm{S}}:=\sum_{n=1}^{m} \int_{t^{n-1}}^{t^{n}}\left|\left\langle\boldsymbol{\sigma}_{t}(t), \hat{\mathbf{e}}(t)\right\rangle\right| d t$,

$\mathcal{J}_{m}^{\mathrm{D}}:=\sum_{n=1}^{m} \int_{t^{n-1}}^{t^{n}}\left|\left\langle P_{0}^{n} \mathbf{f}^{n}-\mathbf{f}(t), \hat{\mathbf{e}}\right\rangle\right| d t, \quad \mathcal{J}_{m}^{\mathrm{C}}:=\sum_{n=1}^{m} \int_{t^{n-1}}^{t^{n}} k_{n}^{-1}\left\langle\Pi^{n} \mathbf{U}^{n-1}-\mathbf{U}^{n-1}, \hat{\mathbf{e}}(t)\right\rangle \mid d t$.

Proof. Take $\hat{\mathbf{e}}$ as a test function in Eq. (4.2) and recall that $\hat{\mathbf{e}} \in \mathbf{Z}$.

Throughout the rest of this paragraph we denote by $t_{\star}^{m} \in\left[0, t^{m}\right]$ the time for which

$$
\left\|\hat{\mathbf{e}}\left(t_{\star}^{m}\right)\right\|=\max _{t \in\left[0, t^{m}\right]}\|\hat{\mathbf{e}}(t)\| .
$$

First an upper bound for the term $\mathcal{J}_{m}^{\mathrm{T}}$ appearing in Lemma 4.3, measuring the local time discretization error, is shown.

Lemma 4.4 (Time error estimate). Let the time estimator $\mathcal{E}_{m}^{\mathrm{T}}$ be given by

$$
\mathcal{E}_{m}^{\mathrm{T}}:=\sum_{n=1}^{m} k_{n} \vartheta_{n} \quad \text { with } \quad \vartheta_{n}:=\frac{k_{n}}{2}\left\|\partial\left(P_{0}^{n} \mathbf{f}^{n}-\bar{\partial} \mathbf{U}^{n}\right)\right\|
$$

Then we have

$$
\mathcal{J}_{m}^{\mathrm{T}} \leq\left\|\hat{\mathbf{e}}\left(t_{\star}^{m}\right)\right\| \mathcal{E}_{m}^{\mathrm{T}}
$$

Proof. We observe that

$$
\int_{t^{n-1}}^{t^{n}}\left|a\left(\hat{\mathbf{U}}(t)-\mathcal{R}_{u}^{n}\left(\mathbf{U}^{n}, P^{n}\right), \hat{\mathbf{e}}(t)\right)\right| d t=\int_{t^{n-1}}^{t^{n}} l_{0}^{n}(t)\left|a\left(\mathcal{R}_{u}^{n-1}\left(\mathbf{U}^{n-1}, P^{n-1}\right)-\mathcal{R}_{u}^{n}\left(\mathbf{U}^{n}, P^{n}\right), \hat{\mathbf{e}}(t)\right)\right| d t .
$$

Since $\hat{\mathbf{e}} \in \mathbf{Z}$, the definition of the reconstruction (3.1) directly yields

$$
\begin{aligned}
& \int_{t^{n-1}}^{t^{n}}\left|a\left(\hat{\mathbf{U}}(t)-\mathcal{R}_{u}^{n}\left(\mathbf{U}^{n}, P^{n}\right), \hat{\mathbf{e}}(t)\right)\right| d t \\
& \quad \leq \int_{t^{n-1}}^{t^{n}} l_{0}^{n}(t)\left\|\Delta_{h}^{n} \mathbf{U}^{n}-B_{h}^{n} P^{n}-\Delta_{h}^{n-1} \mathbf{U}^{n-1}+B_{h}^{n-1} P^{n-1}\right\|\|\hat{\mathbf{e}}(t)\| d t
\end{aligned}
$$

According to (2.25), we have

$$
\begin{aligned}
\mathcal{J}_{m}^{\mathrm{T}} & \leq\left\|\hat{\mathbf{e}}\left(t_{\star}^{m}\right)\right\| \sum_{n=1}^{m} \frac{k_{n}}{2}\left\|\bar{\partial} \mathbf{U}^{n-1}-\bar{\partial} \mathbf{U}^{n}+P_{0}^{n} \mathbf{f}^{n}-P_{0}^{n-1} \mathbf{f}^{n-1}\right\| \\
& =\left\|\hat{\mathbf{e}}\left(t_{\star}^{m}\right)\right\| \sum_{n=1}^{m} \frac{k_{n}^{2}}{2}\left\|\partial\left(P_{0}^{n} \mathbf{f}^{n}-\bar{\partial} \mathbf{U}^{n}\right)\right\| .
\end{aligned}
$$

Next, the term $J_{m}^{\mathrm{S}}$, which accounts for the spatial error, is estimated. 
Lemma 4.5 (Spatial error estimate). Let the estimator $\mathcal{E}_{m}^{\mathrm{S}}$ be defined as

$$
\mathcal{E}_{m}^{\mathrm{S}}:=\sum_{n=1}^{m} k_{n} \delta_{n}:=\sum_{n=1}^{m} k_{n} \eta_{u, \partial}\left(\partial \mathbf{U}^{n}, \partial P^{n}\right) .
$$

Then the following estimate holds

$$
\mathcal{J}_{m}^{\mathrm{S}} \leq\left\|\hat{\mathbf{e}}\left(t_{\star}^{m}\right)\right\| \mathcal{E}_{m}^{\mathrm{S}}
$$

Proof. Noticing that

$$
\boldsymbol{\sigma}_{t}(t)=\frac{\left(\mathcal{R}_{u}^{n}-I\right)\left(\mathbf{U}^{n}, P^{n}\right)-\left(\mathcal{R}_{u}^{n-1}-I\right)\left(\mathbf{U}^{n-1}, P^{n-1}\right)}{k_{n}},
$$

the assertion follows from Assumption 2.

Upper bounds for the term $\mathcal{J}_{m}^{\mathrm{D}}$, which measures the data approximation error, are shown in the next lemma.

Lemma 4.6 (Data error estimate). Let

$$
\zeta_{n}:=\frac{1}{k_{n}} \int_{t^{n-1}}^{t^{n}}\left\|\mathbf{f}(s)-\mathbf{f}^{n}\right\| d t
$$

Then it holds

$$
\mathcal{J}_{m}^{\mathrm{D}} \leq\left\|\hat{\mathbf{e}}\left(t_{\star}^{m}\right)\right\| \sum_{n=1}^{m} k_{n} \zeta_{n}+\sum_{n=1}^{m}\left(\int_{t^{n-1}}^{t^{n}}|\hat{\mathbf{e}}(s)|_{1}^{2}\right)^{1 / 2} k_{n}^{1 / 2}\left\|\left(I-P_{0}^{n}\right) \mathbf{f}^{n}\right\|_{\mathbf{V}^{\star}} .
$$

Proof.

$$
\begin{aligned}
\mathcal{J}_{m}^{\mathrm{D}} & =\sum_{n=1}^{m} \int_{t^{n-1}}^{t^{n}}\left|\left\langle P_{0}^{n} \mathbf{f}^{n}-\mathbf{f}(t), \hat{\mathbf{e}}(t)\right\rangle\right| d t \\
& \leq \sum_{n=1}^{m} \int_{t^{n-1}}^{t^{n}}\left|\left\langle P_{0}^{n} \mathbf{f}^{n}-\mathbf{f}^{n}, \hat{\mathbf{e}}(t)\right\rangle\right|+\left|\left\langle\mathbf{f}^{n}-\mathbf{f}(t), \hat{\mathbf{e}}(t)\right\rangle\right| d t \\
& \leq \sum_{n=1}^{m} \int_{t^{n-1}}^{t^{n}}\left\|\left(I-P_{0}^{n}\right) \mathbf{f}^{n}\right\|_{\mathbf{V}^{\star}}|\hat{\mathbf{e}}(s)|_{1}+\left|\left\langle\mathbf{f}^{n}-\mathbf{f}(t), \hat{\mathbf{e}}(t)\right\rangle\right| d t .
\end{aligned}
$$

Remark 4.7. For e.g. the Crouzeix-Raviart element the term $k_{n}^{1 / 2}\left\|\left(I-P_{0}^{n}\right) \mathbf{f}^{n}\right\|_{\mathbf{V}^{\star}}$ can be further bounded by

$$
\left\|\left(I-P_{0}^{n}\right) \mathbf{f}^{n}\right\|_{\mathbf{V}^{\star}} \leq C\left\|h_{n}\left(I-P_{0}^{n}\right) \mathbf{f}^{n}\right\| .
$$

Indeed, using the orthogonality property of $P_{0}^{n}$, for any $\varphi \in \mathbf{V}$ we obtain

$$
\left\langle\left(I-P_{0}^{n}\right) \mathbf{f}^{n}, \boldsymbol{\varphi}\right\rangle=\left\langle\left(I-P_{0}^{n}\right) \mathbf{f}^{n},\left(\boldsymbol{\varphi}-\mathbf{I}_{h}^{n} \boldsymbol{\varphi}\right)\right\rangle \leq C\left\|h_{n}\left(I-P_{0}^{n}\right) \mathbf{f}^{n}\right\||\boldsymbol{\varphi}|_{1} .
$$

The term $\mathcal{J}_{m}^{\mathrm{C}}$ in Lemma 4.3 may be bounded as follows 
Lemma 4.8 (Coarsening error estimate). Let $\mathcal{E}_{m}^{\mathrm{C}}$ be the coarsening estimator defined by

$$
\left.\mathcal{E}_{m}^{\mathrm{C}}:=\sum_{n=1}^{m} k_{n} \gamma_{n} \quad \text { with } \quad \gamma_{n}:=\| k_{n}^{-1}\left(\Pi^{n}-I\right) U^{n-1}\right) \| .
$$

Then, it holds

$$
\mathcal{J}_{m}^{\mathrm{C}} \leq\left\|\hat{\mathbf{e}}\left(t_{\star}^{m}\right)\right\| \mathcal{E}_{m}^{\mathrm{C}}
$$

Proof. Obvious.

Lemma $4.9\left(L^{\infty}\left(L^{2}\right)\right.$ a posteriori error bound for the velocity error $\left.\hat{\mathbf{e}}\right)$. For $m=1, \ldots, N$, the following estimate holds

$$
\begin{aligned}
\max _{t \in\left[0, t^{m}\right]}\|\hat{\mathbf{e}}(t)\|+ & \left(\int_{0}^{t^{m}}|\hat{\mathbf{e}}(s)|_{1}^{2} d s\right)^{1 / 2} \leq \sqrt{2}\|\hat{\mathbf{e}}(0)\| \\
& +\left\{\left(\mathcal{E}_{m}^{\mathrm{T}}+\mathcal{E}_{m}^{\mathrm{S}}+\mathcal{E}_{m}^{\mathrm{C}}+\mathcal{E}_{m}^{\mathrm{D}, 1}\right)^{2}+\left(\mathcal{E}_{m}^{\mathrm{D}, 2}\right)^{2}\right\}^{1 / 2}
\end{aligned}
$$

where

$$
\mathcal{E}_{m}^{\mathrm{D}, 1}:=\sum_{n=1}^{m} k_{n} \zeta_{n} \quad \text { and } \quad \mathcal{E}_{m}^{\mathrm{D}, 2}:=\left(\sum_{n=1}^{m} k_{n}\left\|\left(I-P_{0}^{n}\right) \mathbf{f}^{n}\right\|_{\mathbf{V}^{\star}}^{2}\right)^{1 / 2} .
$$

Proof. In view of Lemma 4.3, we can easily show that

$$
\left\|\hat{\mathbf{e}}\left(t_{\star}^{m}\right)\right\|^{2}+\int_{0}^{t^{m}}|\hat{\mathbf{e}}(s)|_{1}^{2} d s \leq 2\|\hat{\mathbf{e}}(0)\|^{2}+2\left(\mathcal{J}_{m}^{\mathrm{T}}+\mathcal{J}_{m}^{\mathrm{S}}+\mathcal{J}_{m}^{\mathrm{D}}+\mathcal{J}_{m}^{\mathrm{C}}\right) .
$$

Thus, by making use of the previous lemmas, we can conclude that

$$
\begin{aligned}
\left\|\hat{\mathbf{e}}\left(t_{\star}^{m}\right)\right\|^{2}+\int_{0}^{t^{m}}|\hat{\mathbf{e}}(s)|_{1}^{2} d s \leq & 2\|\hat{\mathbf{e}}(0)\|^{2}+4\left\|\hat{\mathbf{e}}\left(t_{\star}^{m}\right)\right\| \sum_{n=1}^{m} k_{n}\left(\theta_{n}+\delta_{n}+\gamma_{n}+\zeta_{n}\right) \\
& +4 \sum_{n=1}^{m}\left(\int_{t^{n-1}}^{t^{n}}|\hat{\mathbf{e}}(s)|_{1}^{2}\right)^{1 / 2} k_{n}^{1 / 2}\left\|\left(I-P_{0}^{n}\right) \mathbf{f}^{n}\right\| \mathbf{V}^{\star} .
\end{aligned}
$$

The final estimate is derived by using the following fact: Let $c \in \mathbb{R}$ and $\mathbf{a}=\left(a_{0}, a_{1}, \ldots, a_{m}\right), \mathbf{b}=$ $\left(b_{0}, b_{1}, \ldots, b_{m}\right) \in \mathbb{R}^{m+1}$ be such that $|\mathbf{a}|^{2} \leq c^{2}+\mathbf{a} \cdot \mathbf{b}$, then $|\mathbf{a}| \leq|c|+|\mathbf{b}|$. Applying the above result to the case

$$
\begin{aligned}
c=\sqrt{2}\|\hat{\mathbf{e}}(0)\|, \quad a_{0}=\left\|\hat{\mathbf{e}}\left(t_{\star}^{m}\right)\right\|, & a_{n}=\left(\int_{t^{n-1}}^{t^{n}}|\hat{\mathbf{e}}(s)|_{1}^{2} d s\right)^{1 / 2}, n=1, \ldots, m, \\
b_{0}=4 \sum_{n=1}^{m} k_{n}\left(\theta_{n}+\delta_{n}+\gamma_{n}+\zeta_{n}\right), & b_{n}=4 k_{n}^{1 / 2}\left\|\left(I-P_{0}^{n}\right) \mathbf{f}^{n}\right\|_{\mathbf{V}^{\star}}, n=1, \ldots, m,
\end{aligned}
$$

proves the assertion.

Theorem 4.1 $\left(L^{\infty}\left(L^{2}\right)\right.$ a posteriori error estimate for the velocity). For $m=1, \ldots, N$, the following estimate holds

$$
\begin{aligned}
\max _{t \in\left[0, t^{m}\right]}\|\mathbf{u}(t)-\mathbf{U}(t)\| \leq & \sqrt{2}\left\|\mathbf{U}^{0}-\mathcal{R}_{u}^{0} \mathbf{U}^{0}\right\|+\sqrt{2}\left\|\mathbf{U}^{0}-\mathbf{u}_{0}\right\|+\mathcal{E}_{m}^{\mathrm{ell}} \\
& +\left\{\left(\mathcal{E}_{m}^{\mathrm{T}}+\mathcal{E}_{m}^{\mathrm{S}}+\mathcal{E}_{m}^{\mathrm{C}}+\mathcal{E}_{m}^{\mathrm{D}, 1}\right)^{2}+\left(\mathcal{E}_{m}^{\mathrm{D}, 2}\right)^{2}\right\}^{1 / 2} .
\end{aligned}
$$


Proof. The result is a direct consequence of the relation $\mathbf{e}=\hat{\mathbf{e}}-\boldsymbol{\sigma}$, the triangular inequality, Lemma 4.9 and the bound on $\boldsymbol{\sigma}$, Lemma 4.1.

4.3. A posteriori estimates for the pressure. First, we need an estimate for the time derivative $\hat{\mathbf{e}}_{t}$.

Lemma 4.10 (A posteriori estimate for $\hat{\mathbf{e}}_{t}$ ). It holds

$$
\max _{t \in\left[0, t^{m}\right]}\left\{\|\nabla \hat{\mathbf{e}}(t)\|^{2}+\int_{0}^{t}\left\|\hat{\mathbf{e}}_{t}(s)\right\|^{2} d s\right\} \leq\|\nabla \hat{\mathbf{e}}(0)\|^{2}+\tilde{\mathcal{J}}_{m}^{\mathrm{T}}+\tilde{\mathcal{J}}_{m}^{\mathrm{S}}+\tilde{\mathcal{J}}_{m}^{\mathrm{D}}+\tilde{\mathcal{J}}_{m}^{\mathrm{C}},
$$

where $\tilde{\mathcal{J}}_{m}^{\mathrm{T}}, \tilde{\mathcal{J}}_{m}^{\mathrm{S}}, \tilde{\mathcal{J}}_{m}^{\mathrm{D}}, \tilde{\mathcal{J}}_{m}^{\mathrm{C}}$ are defined as in Lemma 4.3 but with $\hat{\mathbf{e}}$ replaced by $\hat{\mathbf{e}}_{t}$.

Proof. Take $\hat{\mathbf{e}}_{t}$ as a test function in Eq. (4.2). The rest follows as in Lemma 4.3.

Lemma 4.11 (Estimate for $\hat{\varepsilon}$ ). There is a constant $C=C\left(\beta, t^{m}\right), \beta$ from Eq. (2.9), such that $(4.28)$

$\int_{0}^{t^{m}}\|\hat{\varepsilon}(t)\|^{2} d t \leq C\left(\|\nabla \hat{\mathbf{e}}(0)\|^{2}+\sum_{n=1}^{m} k_{n}\left(\vartheta_{n}^{2}+\eta_{u, \partial}\left(\partial \mathbf{U}^{n}, \partial P^{n}\right)^{2}+\zeta_{n}^{2}+k_{n}\left\|\left(I-P_{0}^{n}\right) \mathbf{f}^{n}\right\|_{\mathbf{V}^{\star}}^{2}+\gamma_{n}^{2}\right)\right)$. where $\hat{\varepsilon}(t):=\mathcal{R}_{p}^{n}\left(\mathbf{U}^{n}, P^{n}\right)-p(t)$ for $t \in I_{n}$.

Proof. Using the error equation (4.2) one infers for $t \in I_{n}$ and $\varphi \in \mathbf{V}$

$$
\begin{aligned}
b(\boldsymbol{\varphi}, \hat{\varepsilon}(t))= & \left\langle\boldsymbol{\sigma}_{t}(t), \boldsymbol{\varphi}\right\rangle+a\left(\hat{\mathbf{U}}(t)-\mathcal{R}_{u}^{n}\left(\mathbf{U}^{n}, P^{n}\right), \boldsymbol{\varphi}\right)+\left\langle\frac{\Pi^{n} \mathbf{U}^{n-1}-\mathbf{U}^{n-1}}{k_{n}}, \boldsymbol{\varphi}\right\rangle+\left\langle P_{0}^{n} \mathbf{f}^{n}-\mathbf{f}(t), \boldsymbol{\varphi}\right\rangle \\
& -\left\langle\hat{\mathbf{e}}_{t}, \boldsymbol{\varphi}\right\rangle-a(\hat{\mathbf{e}}(t), \boldsymbol{\varphi})=:\langle A(t), \boldsymbol{\varphi}\rangle-a(\hat{\mathbf{e}}(t), \boldsymbol{\varphi}) .
\end{aligned}
$$

By the continuous inf-sup condition $(2.9), \hat{\varepsilon}(t)$ can be bounded by

$$
\beta\|\hat{\varepsilon}(t)\| \leq \sup _{\|\nabla \boldsymbol{\varphi}\|=1}|\langle A(t), \boldsymbol{\varphi}\rangle-a(\hat{\mathbf{e}}(t), \boldsymbol{\varphi})| \leq C(\|A(t)\|+\|\nabla \hat{\mathbf{e}}(t)\|) .
$$

Squaring the above inequality, integrating from $t=0$ to $t=t^{m}$ yields

$$
\int_{0}^{t^{m}}\|\hat{\varepsilon}(t)\|^{2} d t \leq C \int_{0}^{t^{m}}\|A(t)\|^{2}+\|\nabla \hat{\mathbf{e}}(t)\|^{2} d t
$$

The first four terms appearing in $A$ can be estimated like in Lemmas $4.4-4.8, \int_{0}^{t^{m}}\left\|\hat{\mathbf{e}}_{t}(t)\right\|^{2} d t$ by Lemma 4.10 and finally $\int_{0}^{t^{m}}\|\nabla \hat{\mathbf{e}}(t)\|^{2} d t$ by Lemma 4.9 .

Theorem 4.2 (Pressure estimate). There is a generic constant $C>0$ such that the pressure error is bounded by a posteriori terms as follows:

$$
\begin{aligned}
\sum_{n=1}^{m} \int_{t^{n-1}}^{t^{n}}\left\|P^{n}-p(t)\right\|^{2} & \leq C\left(\|\nabla \hat{\mathbf{e}}(0)\|^{2}+\sum_{n=1}^{m} k_{n}\left(\vartheta_{n}^{2}+\zeta_{n}^{2}+k_{n}\left\|\left(I-P_{0}^{n}\right) \mathbf{f}^{n}\right\|_{\mathbf{V}^{\star}}^{2}+\gamma_{n}^{2}\right)\right. \\
& \left.+\sum_{n=1}^{m} k_{n}\left(\eta_{u, \partial}\left(\partial \mathbf{U}^{n}, \partial P^{n}\right)^{2}+\eta_{p}\left(\mathbf{U}^{n}, P^{n}\right)^{2}\right)\right) .
\end{aligned}
$$

Proof. Recall $P^{n}-p(t)=P^{n}-\mathcal{R}_{p}^{n}\left(\mathbf{U}^{n}, P^{n}\right)+\mathcal{R}_{p}^{n}\left(\mathbf{U}^{n}, P^{n}\right)-p(t)=P^{n}-\mathcal{R}_{p}^{n}\left(\mathbf{U}^{n}, P^{n}\right)+\hat{\varepsilon}(t)$ for $t \in I_{n}$. Then use the triangular inequality, Lemma 4.11 and Assumption 2. 


\section{Estimators for CrouzeiX-Raviart and Taylor-Hood finite element spaces}

5.1. Crouzeix-Raviart Spaces. Let $\left(\mathbf{V}_{h}^{n}, S_{h}^{n}\right)$ be the Crouzeix-Raviart nonconforming finite element space of lowest order, [16], associated with $\mathcal{T}_{n}$, that is

$$
\begin{aligned}
\mathbf{V}_{h}^{n}=\left\{\mathbf{v} \in H:\left.\mathbf{v}\right|_{K} \in \mathbb{P}_{1} \times \mathbb{P}_{1} \forall K \in \mathcal{T}_{n}, \mathbf{v}\right. \text { continuous at the midpoints } \\
\text { of } \left.e \in \Sigma_{n} \text { and } 0 \text { at the midpoints of } e \in \mathcal{E}_{n} \cap \partial \Omega\right\}
\end{aligned}
$$

and

$$
S_{h}^{n}=\left\{\psi \in S:\left.\psi\right|_{K} \in \mathbb{P}_{0}, \forall K \in \mathcal{T}_{n}\right\}
$$

where $\mathbb{P}_{l}$ is the space of polynomials in $d$ variables of degree at most $l$.

The finite element approximation $\left(\mathbf{w}_{n}, q_{n}\right) \in \mathbf{V}_{h}^{n} \times S_{h}^{n}$ of the steady-state is defined by Eq. (3.13). It is well known, that the bilinear forms $a(\cdot, \cdot)$ and $b(\cdot, \cdot)$, defined in the elementwise sense, satisfy Eqs. (2.12) and (2.13), see e.g. [16, 32]. A posteriori error analysis of the CrouzeixRaviart nonconforming finite element approximations to the stationary Stokes equations has been considered in, e.g. [17] (estimates in the energy norm) or [24] (for the $L^{2}$ norm). For corresponding estimates in the finite volume case see [15]. For completeness we present a proof for the existence of an estimator for the $L^{2}$-norm of the velocity including mesh changes.

Lemma 5.1. There are interpolation operators $I_{h}^{n}:=H_{0}^{1} \rightarrow S_{h}^{n}$ and $\mathbf{I}_{h}^{n}:=\mathbf{V} \rightarrow \mathbf{V}_{h}^{n} \cap V$ satisfying the following interpolation estimates:

$$
\begin{aligned}
& \left\|\phi-I_{h}^{n} \psi\right\|_{0, K} \leq C h(K)|\phi|_{1, K} \\
& \left\|\phi-I_{h}^{n} \phi\right\|_{0, e} \leq C h(e)^{1 / 2}|\phi|_{1, \tilde{K}} \\
& \left\|\boldsymbol{\varphi}-\mathbf{I}_{h}^{n} \boldsymbol{\varphi}\right\|_{0, K} \leq C h(K)^{j}|\boldsymbol{\varphi}|_{j, \tilde{K}} \quad j=1,2 \\
& \left\|\boldsymbol{\varphi}-\mathbf{I}_{h}^{n} \boldsymbol{\varphi}\right\|_{0, e} \leq C h(K)^{j-1 / 2}|\boldsymbol{\varphi}|_{j, \tilde{K}} \quad j=1,2
\end{aligned}
$$

with $\tilde{K}=\cup_{K \cap \bar{K} \neq \emptyset} \bar{K}$ and $h(K)=h(x)$ for $x \in K, h(e)=h(x)$ for $x \in e$.

Proof. See $[16,32]$.

Before we proceed with an elliptic error estimate, some notation is introduced. Denote by $\mathbf{R}_{K}^{n}, K \in \mathcal{T}_{n}$ the inner residual given by

$$
\mathbf{R}_{K}^{n}\left(\mathbf{w}_{n}, q_{n}\right):=\left.\left(-\Delta \mathbf{w}_{n}+\nabla q_{n}-\mathbf{h}\right)\right|_{K}=-\left.\mathbf{h}\right|_{K}
$$

with $\mathbf{h}=-\Delta_{h}^{n} \mathbf{w}_{n}+B_{h}^{n} q_{n}$. Note that if $\left(\mathbf{w}_{n}, q_{n}\right)=\left(\mathbf{U}^{n}, P^{n}\right)$, i.e. is the discrete solution, then $\mathbf{h}$ can equivalently be written as $\mathbf{h}=P_{0}^{n} \mathbf{f}^{n}-k_{n}^{-1}\left(\mathbf{U}^{n}-\Pi^{n} \mathbf{U}^{n-1}\right)$.

For all edges $e \in \Sigma_{n}$ define

$$
\mathbf{J}_{e, \mathbf{n}}\left(\mathbf{w}_{n}, q_{n}\right)=\left\{\begin{array}{ll}
\llbracket\left(\nabla \mathbf{w}_{n}-q_{n} \mathbf{I}\right) \mathbf{n}_{e} \rrbracket_{e}, & \text { if } e \in \Sigma_{n}, \\
0, & \text { otherwise, }
\end{array} \quad \mathbf{J}_{e, \boldsymbol{\tau}}\left(\mathbf{w}_{n}\right)= \begin{cases}\llbracket \nabla \mathbf{w}_{n} \boldsymbol{\tau}_{e} \rrbracket_{e}, & \text { if } e \in \Sigma_{n}, \\
2 \nabla \mathbf{w}_{n} \boldsymbol{\tau}_{e}, & \text { otherwise }\end{cases}\right.
$$

where $\boldsymbol{\tau}_{e}:=\left[-n_{2}, n_{1}\right]^{T}$ is a unit tangential vector on $e$ with fixed but arbitrary orientation.

At this point it is useful to note that the term involving $\mathbf{J}_{e, \boldsymbol{\tau}}$ can be replaced by the jump of $\mathbf{w}_{n}$. Indeed, an elementary calculation shows that, [24],

$$
\left\|\llbracket \mathbf{w}_{n} \rrbracket_{e}\right\|_{0, e}^{2}=\frac{h_{e}^{2}}{12}\left\|\mathbf{J}_{e, \boldsymbol{\tau}}\left(\mathbf{w}_{n}\right)\right\|_{0, e}^{2} .
$$


Lemma 5.2 ( $L^{2}$-error estimate for the velocity). There is a generic constant $C>0$ such that for given $\left(\mathbf{w}_{n-1}, q_{n-1}\right) \in \mathbf{Z}_{h}^{n-1} \times S_{h}^{n-1}$ and $\left(\mathbf{w}_{n}, q_{n}\right) \in \mathbf{Z}_{h}^{n} \times S_{h}^{n}$

$$
\left\|\partial\left(I-\mathcal{R}_{u}^{n}\right)\left(\mathbf{w}_{n}, q_{n}\right)\right\|^{2} \leq C \eta_{u, \partial}\left(\partial\left(\mathbf{w}_{n}, q_{n}\right)\right)
$$

with $\eta_{u, \partial}$ defined by

$$
\eta_{u, \partial}\left(\partial\left(\mathbf{w}_{n}, q_{n}\right)\right)^{2}:=\left\|\hat{h}_{n}^{2} \mathbf{R}_{K}^{n}\left(\partial \mathbf{w}_{n}, \partial q_{n}\right)\right\|_{\check{\mathcal{T}}_{n}}^{2}+\left\|\hat{h}_{e}^{3 / 2} \mathbf{J}_{e, \mathbf{n}}\left(\partial \mathbf{w}_{n}, \partial q_{n}\right)\right\|_{\check{\Sigma}_{n}}^{2}+\left\|\hat{h}_{e}^{3 / 2} \mathbf{J}_{e, \boldsymbol{\tau}}\left(\partial \mathbf{w}_{n}\right)\right\|_{\Sigma_{n}}^{2} .
$$

Further,

$$
\left\|\left(I-\mathcal{R}_{u}^{n}\right)\left(\mathbf{w}_{n}, q_{n}\right)\right\|^{2} \leq C \eta_{u}\left(\mathbf{w}_{n}, q_{n}\right)
$$

with $\eta_{u}$ defined by

$$
\eta_{u}\left(\mathbf{w}_{n}, q_{n}\right)^{2}:=\left\|h_{n}^{2} \mathbf{R}_{K}^{n}\left(\mathbf{w}_{n}, q_{n}\right)\right\|_{\mathcal{T}_{n}}^{2}+\left\|h_{e}^{3 / 2} \mathbf{J}_{e, \mathbf{n}}\left(\mathbf{w}_{n}, q_{n}\right)\right\|_{\Sigma_{n}}^{2}+\left\|h_{e}^{3 / 2} \mathbf{J}_{e, \boldsymbol{\tau}}\left(\partial \mathbf{w}_{n}\right)\right\|_{\Sigma_{n}}^{2} .
$$

Proof. Introducing the classical conforming space

$$
\mathbf{X}_{h}^{n}:=\mathbf{V} \cap \mathbf{V}_{h}^{n} \cap \mathbf{V}_{h}^{n-1},
$$

the following orthogonality relation holds

$$
a\left(\mathbf{g}, \hat{\boldsymbol{\varphi}}_{n}\right)+b\left(\hat{\boldsymbol{\varphi}}_{n}, \xi\right)=0 \quad \forall \hat{\boldsymbol{\varphi}}_{n} \in \mathbf{X}_{h}^{n}
$$

where $(\mathbf{g}, \xi)=\partial\left(I-\mathcal{R}^{n}\right)\left(\mathbf{w}_{n}, q_{n}\right)$.

Consider the dual problem: find $(\mathbf{z}, s) \in\left(\mathbf{H}^{2}(\Omega) \cap \mathbf{H}_{0}^{1}(\Omega) \times H^{1}(\Omega) \cap L_{0}^{2}(\Omega)\right)$ such that

$$
\begin{aligned}
& -\Delta \mathbf{z}-\nabla s=\mathbf{g} \quad \text { in } \Omega, \\
& \operatorname{div} \mathbf{z}=0 \quad \text { in } \Omega, \\
& \mathbf{z}=\mathbf{0} \text { on } \partial \Omega,
\end{aligned}
$$

fulfilling the regulartiy estimate

$$
\|\mathbf{z}\|_{2}+\|s\|_{1} \leq C\|\mathbf{g}\|
$$

Integrating by parts gives

$$
\|\mathbf{g}\|^{2}=\langle-\Delta \mathbf{z}-\nabla s, \mathbf{g}\rangle=\sum_{K \in \check{\mathcal{T}}_{n}}\left\{\int_{K}\left(\nabla \mathbf{z}: \nabla \mathbf{g}+s \operatorname{div} \mathbf{g}-\int_{\partial K} \nabla \mathbf{z} \mathbf{n} \cdot \partial \mathbf{w}_{n}-\int_{\partial K} s \mathbf{n} \cdot \partial \mathbf{w}_{n}\right\} .\right.
$$

Since $\operatorname{div} \mathbf{z}=0$ relation (5.8) may be written as follows

$$
\|\mathbf{g}\|^{2}=\sum_{K \in \check{\mathcal{T}}_{n}}\left\{\int_{K}(\nabla \mathbf{z}: \nabla \mathbf{g}+s \operatorname{div} \mathbf{g}-\xi \operatorname{div} \mathbf{z})-\int_{\partial K} \nabla \mathbf{z} \mathbf{n} \cdot \partial \mathbf{w}_{n}-\int_{\partial K} s \mathbf{n} \cdot \partial \mathbf{w}_{n}\right\} .
$$

Using the orthogonality property (5.5) and the fact that $b\left(\mathbf{g}, \hat{\phi}_{n}\right)=0 \forall \hat{\phi}_{n} \in S_{h}^{n} \cap S_{h}^{n-1}$, we have

$$
\begin{aligned}
\|\mathbf{g}\|^{2}= & \sum_{K \in \check{\mathcal{T}}_{n}}\left\{\int_{K}(-\Delta \mathbf{g}+\nabla \xi) \cdot\left(\mathbf{z}-\hat{\boldsymbol{\varphi}}_{n}\right)\right. \\
& \left.+\int_{K}\left(s-\hat{\phi}_{n}\right) \operatorname{div} \partial \mathbf{w}_{n}+\int_{\partial K}\left(\nabla \partial \mathbf{w}_{n}-\partial q_{n} I\right) \mathbf{n} \cdot\left(\mathbf{z}-\hat{\boldsymbol{\varphi}}_{n}\right)-\int_{\partial K}(\nabla \mathbf{z} \mathbf{n}+s \mathbf{n}) \cdot \partial \mathbf{w}_{n}\right\}
\end{aligned}
$$

for all $\hat{\varphi}_{n} \in \mathbf{X}_{h}^{n}$. 
Note that since $\mathbf{w}_{n-1}, \mathbf{w}_{n}$ are piecewise linear and the pressures are piecewise constant it holds $\left(\operatorname{div} \partial \mathbf{w}_{n}\right)_{\mid K}=0$. Then one gets the identity

$$
\begin{aligned}
\|\mathbf{g}\|^{2}= & \sum_{K \in \check{T}_{n}}\left\{\int_{K}-\mathbf{R}_{K}^{n}\left(\partial \mathbf{w}_{n}, \partial q_{n}\right) \cdot\left(\mathbf{z}-\hat{\boldsymbol{\varphi}}_{n}\right)\right. \\
& \left.+\int_{\partial K}\left(\nabla \partial \mathbf{w}_{n}-\partial q_{n} I\right) \mathbf{n} \cdot\left(\mathbf{z}-\hat{\boldsymbol{\varphi}}_{n}\right)-\int_{\partial K}(\nabla \mathbf{z} \mathbf{n}+s \mathbf{n}) \cdot \partial \mathbf{w}_{n}\right\} .
\end{aligned}
$$

Let $\hat{\boldsymbol{\varphi}}_{n}:=\hat{\mathbf{I}}_{h}^{n} \mathbf{z} \in \mathbf{X}_{h}^{n}$ and $\hat{\phi}_{n}:=\hat{I}_{h}^{n} s \in S_{h}^{n} \cap S_{h}^{n-1}$ be the respective interpolants of $\mathbf{z}$ and $s$. Then the terms in Eq. (5.10) can be estimated using the approximation properties Lemma 5.1 and the regularity estimate (5.7) as

$$
\sum_{K \in \check{T}_{n}} \int_{K}-\mathbf{R}_{K}^{n}\left(\partial \mathbf{w}_{n}, \partial q_{n}\right) \cdot\left(\mathbf{z}-\hat{\mathbf{I}}_{h}^{n} \mathbf{z}\right) \leq\left(\sum_{K \in \check{T}_{n}} \hat{h}_{n}^{4}\left\|\mathbf{R}_{K}^{n}\left(\partial \mathbf{w}_{n}, \partial q_{n}\right)\right\|_{0, K}^{2}\right)^{1 / 2}\|\mathbf{g}\|,
$$

$$
\sum_{K \in \check{T}_{n}} \int_{\partial K}\left(\nabla \partial \mathbf{w}_{n}-\partial q_{n}\right) \mathbf{n} \cdot\left(\mathbf{z}-\hat{\mathbf{I}}_{h}^{n} \mathbf{z}\right) \leq C\left(\sum_{e \in \check{\Sigma}_{n}} \hat{h}_{e}^{3}\left\|\mathbf{J}_{e, \mathbf{n}}^{n}\left(\partial \mathbf{w}_{n}, \partial q_{n}\right)\right\|_{0, e}^{2}\right)^{1 / 2}\|\mathbf{g}\|
$$

and

$$
\sum_{K \in \check{T}_{n}} \int_{\partial K}(\nabla \mathbf{z} \mathbf{n}+s \mathbf{n}) \cdot \partial \mathbf{w}_{n} \leq C\left(\sum_{e \in \check{\Sigma}_{n}} \hat{h}_{e}^{3}\left\|\mathbf{J}_{e, \boldsymbol{\tau}}^{n}\left(\partial \mathbf{w}_{n}\right)\right\|_{0, e}^{2}\right)^{1 / 2}\|\mathbf{g}\| .
$$

To obtain the last bound we notice that

$$
\sum_{K \in \check{T}_{n}} \int_{\partial K} \nabla \mathbf{z} \mathbf{n} \cdot \partial \mathbf{w}_{n}=\sum_{e \in \check{\Sigma}_{n}} \int_{e} \nabla \mathbf{z} \mathbf{n} \cdot \llbracket \partial \mathbf{w}_{n} \rrbracket .
$$

Assume for a moment that for $m_{e}$ denoting the middle of the edge $e$, we have $\llbracket \partial \mathbf{w}_{n} \rrbracket\left(m_{e}\right)=0$. Then,

$$
\sum_{e \in \check{\Sigma}_{n}} \int_{e} \llbracket \partial \mathbf{w}_{n} \rrbracket=0
$$

since $\partial \mathbf{w}_{n}$ is linear on each $e$. Hence,

$$
\sum_{K \in \check{T}_{n}} \int_{\partial K} \nabla \mathbf{z} \mathbf{n} \cdot \partial \mathbf{w}_{n}=\sum_{e \in \check{\Sigma}_{n}} \int_{e} \nabla\left[\mathbf{z}-\hat{\mathbf{I}}_{h}^{n} \mathbf{z}\right] \mathbf{n} \cdot \llbracket \partial \mathbf{w}_{n} \rrbracket .
$$

In view of (5.1) and (5.3), the last identity implies that this term is bounded by the upper bound in (5.13). Notice now that $\llbracket \partial \mathbf{w}_{n} \rrbracket\left(m_{e}\right)=0$ holds in most cases, since $\mathbf{w}_{n}$ or $\mathbf{w}_{n-1}$ will be either continuous on $e$ or continuous only at $m_{e}$ depending on whether $e$ is an edge of the corresponding space. The only case where $\llbracket \partial \mathbf{w}_{n} \rrbracket\left(m_{e}\right)=0$ does not hold is when the side $e$ is refined or coarsened going from $n-1$ to $n$. But then still, $\int_{\tilde{e}} \llbracket \partial \mathbf{w}_{n} \rrbracket=0$ where $\tilde{e}$ is the union of the the two (or more) possibly refined edges. Then a modification of the above argument concludes the proof. Notice that we do not impose any restrictions on the relationship of two consecutive meshes, we just compare their approximability properties. A similar argument applies to the term in (5.13) involving $s$. 
Lemma 5.3 ( $L^{2}$-error estimate for the pressure). There is a generic constant $C>0$ such that for $\left(\mathbf{w}_{n}, q_{n}\right) \in \mathbf{Z}_{h}^{n} \times S_{h}^{n}$

$$
\left\|\nabla\left(I-\mathcal{R}_{u}^{n}\right)\left(\mathbf{w}_{n}, q_{n}\right)\right\|+\left\|\left(I-\mathcal{R}_{p}^{n}\right)\left(\mathbf{w}_{n}, q_{n}\right)\right\| \leq C \eta_{p}\left(\mathbf{w}_{n}, q_{n}\right)
$$

with $\eta_{p}\left(\mathbf{w}_{n}, q_{n}\right)$ defined by

$$
\eta_{p}\left(\mathbf{w}_{n}, q_{n}\right)^{2}=\left\|h_{n} \mathbf{R}_{K}^{n}\left(\mathbf{w}_{n}, q_{n} ; \mathbf{g}\right)\right\|_{\mathcal{T}_{n}}^{2}+\left\|h_{e}^{1 / 2} \mathbf{J}_{e, \mathbf{n}}\left(\mathbf{w}_{n}, q_{n}\right)\right\|_{\Sigma_{n}}^{2}+\left\|h_{e}^{1 / 2} \mathbf{J}_{e, \tau}\left(\mathbf{w}_{n}\right)\right\|_{\Sigma_{n}}^{2} .
$$

Proof. See [17].

As an immediate consequence of the previous two lemmas one derives:

Corollary 5.1. Assumption 2 is fulfilled with the estimators from Lemmas 5.2, 5.3.

5.2. Taylor-Hood Spaces. Let $\left(\mathbf{V}_{h}^{n}, S_{h}^{n}\right)$ be the Taylor-Hood conforming finite element space, associated with $\mathcal{T}_{n}$, that is, [31],

$$
\mathbf{V}_{h}^{n}=\left\{\mathbf{v} \in H_{0}^{1}(\Omega)^{2}:\left.\mathbf{v}\right|_{K} \in \mathbb{P}_{2} \times \mathbb{P}_{2} \forall K \in \mathcal{T}_{n}\right\}
$$

and

$$
S_{h}^{n}=\left\{\psi \in S:\left.\psi\right|_{K} \in \mathbb{P}_{1}, \forall K \in \mathcal{T}_{n}\right\} .
$$

Notice that higher order Taylor-Hood spaces can be treated using similar arguments.

The finite element approximation $\left(\mathbf{w}_{n}, q_{n}\right) \in \mathbf{V}_{h}^{n} \times S_{h}^{n}$ of the steady-state is defined by Eq. (3.13). It is well known, that the bilinear forms $a(\cdot, \cdot)$ and $b(\cdot, \cdot)$, defined in the elementwise sense, satisfy Eqs. (2.12) and (2.13), see e.g. [32]. Taylor-Hood is a very popular pair for fluid flow computations. The estimators for these elements appear to be known and their derivation is quite similar to the previous section, although simpler, see e.g., [33, 21] and [35]. For completeness we present the main steps of the derivation of the estimator in the $L^{2}$-norm of the velocity including mesh changes.

As before we shall use the following notation for residuals and jumps. Denote by $\mathbf{R}_{K}^{n}, K \in \mathcal{T}_{n}$ the inner residual given by

$$
\mathbf{R}_{K}^{n}\left(\mathbf{w}_{n}, q_{n}\right):=\left.\left(-\Delta \mathbf{w}_{n}+\nabla q_{n}-\mathbf{h}\right)\right|_{K}=-\left.\mathbf{h}\right|_{K} .
$$

For the edges $e \in \Sigma_{n}$ define

$$
\mathbf{J}_{e, \mathbf{n}}\left(\mathbf{w}_{n}, q_{n}\right)= \begin{cases}\llbracket \nabla \mathbf{w}_{n} \mathbf{n}_{e} \rrbracket_{e}, & \text { if } e \in \Sigma_{n}, \\ 0, & \text { otherwise. }\end{cases}
$$

Notice that a tangential jump term is not involved in the estimators. Also, there is no jump of the pressure across interelement boundaries, since the pressure space here consists of globally continuous functions.

Lemma 5.4 ( $L^{2}$-error estimate for the velocity). There is a generic constant $C>0$ such that for given $\left(\mathbf{w}_{n-1}, q_{n-1}\right) \in \mathbf{Z}_{h}^{n-1} \times S_{h}^{n-1}$ and $\left(\mathbf{w}_{n}, q_{n}\right) \in \mathbf{Z}_{h}^{n} \times S_{h}^{n}$

$$
\left\|\partial\left(I-\mathcal{R}_{u}^{n}\right)\left(\mathbf{w}_{n}, q_{n}\right)\right\|^{2} \leq C \eta_{u, \partial}\left(\partial\left(\mathbf{w}_{n}, q_{n}\right)\right)
$$

with $\eta_{u, \partial}$ defined by

$$
\eta_{u, \partial}\left(\partial\left(\mathbf{w}_{n}, q_{n}\right)\right)^{2}:=\left\|\hat{h}_{n}^{2} \mathbf{R}_{K}^{n}\left(\partial \mathbf{w}_{n}, \partial q_{n}\right)\right\|_{\check{\mathcal{T}}_{n}}^{2}+\left\|\hat{h}_{e}^{3 / 2} \mathbf{J}_{e, \mathbf{n}}\left(\partial \mathbf{w}_{n}, \partial q_{n}\right)\right\|_{\check{\Sigma}_{n}}^{2}+\left\|\hat{h}_{n} \operatorname{div} \partial \mathbf{w}_{n}\right\|_{\check{\mathcal{T}}_{n}}^{2} .
$$

Further,

$$
\left\|\left(I-\mathcal{R}_{u}^{n}\right)\left(\mathbf{w}_{n}, q_{n}\right)\right\|^{2} \leq C \eta_{u}\left(\mathbf{w}_{n}, q_{n}\right)
$$


with $\eta_{u}$ defined by

$$
\eta_{u}\left(\mathbf{w}_{n}, q_{n}\right)^{2}:=\left\|h_{n}^{2} \mathbf{R}_{K}^{n}\left(\mathbf{w}_{n}, q_{n}\right)\right\|_{\mathcal{T}_{n}}^{2}+\left\|h_{e}^{3 / 2} \mathbf{J}_{e, \mathbf{n}}\left(\mathbf{w}_{n}, q_{n}\right)\right\|_{\Sigma_{n}}^{2}+\left\|h_{n} \operatorname{div} \partial \mathbf{w}_{n}\right\|_{\mathcal{T}_{n}}^{2} .
$$

Proof. As before we introduce the conforming space $\mathbf{X}_{h}^{n}:=\mathbf{V}_{h}^{n} \cap \mathbf{V}_{h}^{n-1}$. Using the corresponding notation and arguments similar to Lemma 5.2 we arrive at the identity

$$
\begin{aligned}
\|\mathbf{g}\|^{2}= & \sum_{K \in \check{T}_{n}}\left\{\int_{K}-\mathbf{R}_{K}^{n}\left(\partial \mathbf{w}_{n}, \partial q_{n}\right) \cdot\left(\mathbf{z}-\hat{\boldsymbol{\varphi}}_{n}\right)+\int_{K}\left(s-\hat{\phi}_{n}\right) \operatorname{div} \partial \mathbf{w}_{n}\right. \\
& \left.+\int_{\partial K}\left(\nabla \partial \mathbf{w}_{n}-\partial q_{n} I\right) \mathbf{n} \cdot\left(\mathbf{z}-\hat{\boldsymbol{\varphi}}_{n}\right)-\int_{\partial K}(\nabla \mathbf{z} \mathbf{n}+s \mathbf{n}) \cdot \partial \mathbf{w}_{n}\right\} .
\end{aligned}
$$

Notice that the last term is zero due to the conformity of the velocity spaces. In fact,

$$
\sum_{K \in \check{T}_{n}} \int_{\partial K}(\nabla \mathbf{z} \mathbf{n}+s \mathbf{n}) \cdot \partial \mathbf{w}_{n}=\sum_{e \in \check{\Sigma}_{n}} \int_{e}(\nabla \mathbf{z} \mathbf{n}+s \mathbf{n}) \cdot \llbracket \partial \mathbf{w}_{n} \rrbracket=0 .
$$

Now, let $\hat{\varphi}_{n}:=\hat{\mathbf{I}}_{h}^{n} \mathbf{z} \in \mathbf{X}_{h}^{n}$ and $\hat{\phi}_{n}:=\hat{I}_{h}^{n} s \in S_{h}^{n} \cap S_{h}^{n-1}$ be the respective interpolants of $\mathbf{z}$ and $s$. Then the remaining terms in Eq. (5.18) can be estimated as before using Lemma 5.1 and the Stokes regularity (5.7),

$$
\begin{aligned}
& \sum_{K \in \check{T}_{n}} \int_{K}-\mathbf{R}_{K}^{n}\left(\partial \mathbf{w}_{n}, \partial q_{n}\right) \cdot\left(\mathbf{z}-\hat{\mathbf{I}}_{h}^{n} \mathbf{z}\right) \leq\left(\sum_{K \in \check{T}_{n}} \hat{h}_{n}^{4}\left\|\mathbf{R}_{K}^{n}\left(\partial \mathbf{w}_{n}, \partial q_{n}\right)\right\|_{0, K}^{2}\right)^{1 / 2}\|\mathbf{g}\|, \\
& \sum_{K \in \check{T}_{n}} \int_{\partial K}\left(\nabla \partial \mathbf{w}_{n}-\partial q_{n}\right) \mathbf{n} \cdot\left(\mathbf{z}-\hat{\mathbf{I}}_{h}^{n} \mathbf{z}\right) \leq C\left(\sum_{e \in \check{\Sigma}_{n}} \hat{h}_{e}^{3}\left\|\mathbf{J}_{e, \mathbf{n}}^{n}\left(\partial \mathbf{w}_{n}, \partial q_{n}\right)\right\|_{0, e}^{2}\right)^{1 / 2}\|\mathbf{g}\|,
\end{aligned}
$$

and

$$
\sum_{K \in \check{T}_{n}} \int_{K}\left(s-\hat{I}_{h}^{n} s\right) \operatorname{div} \partial \mathbf{w}_{n} \leq C\left(\sum_{K \in \check{T}_{n}} \hat{h}_{n}^{2}\left\|\operatorname{div} \partial \mathbf{w}_{n}\right\|_{0, K}^{2}\right)^{1 / 2}\|\mathbf{g}\|,
$$

and the proof is complete.

For completeness we state the estimate for the pressure.

Lemma 5.5 ( $L^{2}$-error estimate for the pressure). There is a generic constant $C>0$ such that for $\left(\mathbf{w}_{n}, q_{n}\right) \in \mathbf{Z}_{h}^{n} \times S_{h}^{n}$

$$
\left\|\nabla\left(I-\mathcal{R}_{u}^{n}\right)\left(\mathbf{w}_{n}, q_{n}\right)\right\|+\left\|\left(I-\mathcal{R}_{p}^{n}\right)\left(\mathbf{w}_{n}, q_{n}\right)\right\| \leq C \eta_{p}\left(\mathbf{w}_{n}, q_{n}\right)
$$

with $\eta_{p}\left(\mathbf{w}_{n}, q_{n}\right)$ defined by

$$
\eta_{p}\left(\mathbf{w}_{n}, q_{n}\right)^{2}=\left\|h_{n} \mathbf{R}_{K}^{n}\left(\mathbf{w}_{n}, q_{n} ; \mathbf{g}\right)\right\|_{\mathcal{T}_{n}}^{2}+\left\|h_{e}^{1 / 2} \mathbf{J}_{e, \mathbf{n}}\left(\mathbf{w}_{n}, q_{n}\right)\right\|_{\Sigma_{n}}^{2}+\left\|\operatorname{div} \partial \mathbf{w}_{n}\right\|_{\mathcal{T}_{n}}^{2} .
$$

As an immediate consequence of the previous two lemmas one derives:

Corollary 5.2. When Taylor-Hood finite element spaces are considered Assumption 2 is fulfilled with the estimators from Lemmas 5.4, 5.5. 


\section{Computational Results}

In this section we discuss numerical experiments related to the previous theoretical analysis.

The asymptotic behavior of the error estimators is studied and compared with the true error. For the implementation of the estimators we used FEniCS [3] and NAVIER [5].

We start with two examples for the time-dependent Stokes problem on the unit square $\Omega:=$ ] $0,1\left[^{2}, T=1\right.$. The exact solutions $(\mathbf{u}, p)$ are:

Example 1. $\mathbf{u}_{0}=\mathbf{u}(\cdot, 0)=0$ in $\Omega, \mathbf{u}=0$ on $\left.\partial \Omega \times\right] 0,1[$ and $\operatorname{div} \mathbf{u}=0$ in $\Omega \times] 0,1[$.

$$
\left\{\begin{array}{l}
\mathbf{u}(x, y, t)=\left(t^{2}\left(x^{4}-2 x^{3}+x^{2}\right)\left(4 y^{3}-6 y^{2}+2 y\right), t^{2}\left(4 x^{3}-6 x^{2}+2 x\right)\left(y^{4}-2 y^{3}+y^{2}\right)\right)^{T} \\
p(x, y, t)=t^{2}(x+y) .
\end{array}\right.
$$

Example 2. $\mathbf{u}_{0}=\mathbf{u}(\cdot, 0)=0$ in $\Omega, \mathbf{u}=\mathbf{g} \neq 0$ on $\left.\partial \Omega \times\right] 0,1[$ and $\operatorname{div} \mathbf{u}=0$ in $\Omega \times] 0,1[$.

$$
\left\{\begin{array}{l}
\mathbf{u}(x, y, t)=\sin (t)(\sin (\pi x) \sin (\pi y), \cos (\pi x) \cos (\pi y))^{T}, \\
p(x, y, t)=\sin (t)\left(\sin (\pi x)+\sin (\pi y)-\frac{2}{\pi}\right) .
\end{array}\right.
$$

In both cases the right-hand side $\mathbf{f}$ is calculated by applying the time-dependent Stokes equation to $(\mathbf{u}, p)$.

We conduct tests on uniform meshes with uniform time steps. For the discretization in space we use the nonconforming P1/P0-Crouzeix-Raviart elements and the conforming P2/P1-TaylorHood elements. The computed quantities are: the velocity error in the discrete $L^{\infty}\left(0, t^{m} ; L^{2}(\Omega)\right)$ norm

$$
\max _{0 \leq n \leq m}\left\|\mathbf{e}^{n}\right\|=\max _{0 \leq n \leq m}\left\|\mathbf{u}\left(t^{n}\right)-\mathbf{U}^{n}\right\|,
$$

and the space, time and Stokes reconstruction estimators introduced in the previous section.

The coarsening error estimator $\mathcal{E}_{N}^{\mathrm{C}}$ is excluded from the numerical experiments since it vanishes. Moreover, the terms corresponding to the approximation of data $\mathbf{u}_{0}$ and $\mathbf{f}$ clearly are of optimal order and thus do not contain interesting information for our purposes. Moreover, we compute the total estimator $\mathcal{E}_{m}$ defined as follows

$$
\mathcal{E}_{m}:=\mathcal{E}_{m}^{\mathrm{T}}+\mathcal{E}_{m}^{\mathrm{S}}+\mathcal{E}_{m}^{\mathrm{ell}}, \quad 1 \leq m \leq N .
$$

Then, the corresponding effectivity index is defined as

$$
\mathrm{EI}\left(t^{m}\right):=\frac{\mathcal{E}_{m}}{\max _{0 \leq n \leq m}\left\|\mathbf{e}^{n}\right\|}, \quad 1 \leq m \leq N
$$

For all quantities of interest we look at their experimental order of convergence (EOC), which is defined as follows: for a given finite sequence of successive runs (indexed by $i$ ), the EOC of the corresponding sequence of quantities of interest $E(i)$ (error, estimator or part of an estimator), is itself a sequence defined by

$$
\operatorname{EOC}(E(i))=\frac{\log (E(i+1) / E(i))}{\log (h(i+1) / h(i))}
$$

where $h(i)$ denotes the mesh-size of the run $i$. The values of EOC of a given quantity indicates its order.

Note that, in case that the backward Euler method is combined with finite element spaces that consist of nonconforming $\mathrm{P} 1 / \mathrm{P} 0$ Crouzeix-Raviart elements, we expect the velocity error in $L^{\infty}\left(0, T ; L^{2}(\Omega)\right)$ norm to be $O\left(k+h^{2}\right)$. Therefore, in order to show the optimality of the computed quantities, that is $\mathrm{EOC}=2$, in each run we take $k=h^{2}$. On the other hand, if the backward Euler method is combined with P2/P1 Taylor-Hood elements, we expect the velocity 
error in $L^{\infty}\left(0, T ; L^{2}(\Omega)\right)$ norm to be $O\left(k+h^{3}\right)$. To show the optimality of the $L^{\infty}\left(0, t^{m}, L^{2}(\Omega)\right)$ error norm and of the corresponding estimators, that is $\mathrm{EOC}=3$, in each run we take $k=h^{3}$.

We are also interested in computing the effectivity index defined as the ratio between the total a posteriori error estimator and $L^{\infty}\left(0, t^{m} ; L^{2}(\Omega)\right)$ norm of the error, namely

$$
\mathrm{EI}\left(t^{m}\right):=\frac{\mathcal{E}_{m}}{\max _{0 \leq n \leq m}\left\|\mathbf{e}^{n}\right\|}, \quad 1 \leq m \leq N .
$$

The main conclusion from these two examples is that all error estimators, in both cases of spatial discretization, decrease with optimal order with respect to temporal and spatial variable, see Tables 1-6. The results listed in Table 1, Table 3 and Table 5 show that all effectivity indices are asymptotically constant.

Note that as usual with residual based error estimators the effectivity indices are not close to 1 . However, for a given pair of finite elements they are (asymptotically) constant. For using the estimators in devicing a practical adaptive strategy, the estimators have to be calibrated by some computational examples (like the ones here).

\begin{tabular}{c|cccc}
\hline$h$ & $\max _{n}\left\|\mathrm{e}^{n}\right\|$ & $\mathrm{EOC}$ & $\mathcal{E}_{N}$ & $\mathrm{EI}\left(t^{N}\right)$ \\
\hline 0.50000 & $1.4263 \mathrm{e}-02$ & - & $7.0117 \mathrm{e}-01$ & 49 \\
0.25000 & $4.6766 \mathrm{e}-03$ & 1.61 & $2.0780 \mathrm{e}-01$ & 45 \\
0.12500 & $1.3348 \mathrm{e}-03$ & 1.81 & $5.5285 \mathrm{e}-02$ & 41 \\
0.06250 & $3.5317 \mathrm{e}-04$ & 1.92 & $1.4226 \mathrm{e}-02$ & 40 \\
0.03125 & $9.0092 \mathrm{e}-05$ & 1.97 & $3.5997 \mathrm{e}-03$ & 40
\end{tabular}

TABle 1. Example 1: P1/P0 Crouzeix-Raviart elements combined with the backward Euler. The $L^{\infty}\left(0,1 ; L^{2}(\Omega)\right)$-error for the velocity, the corresponding EOC, the total estimator $\mathcal{E}_{N}$ and the corresponding effectivity index $\operatorname{EI}\left(t^{N}\right)$.

Example 3. With this example we study the effect of mesh modification. The domain here is $\Omega=]-0.5,0.5\left[^{2}, T=1.6\right.$ and the exact solution is

$$
\left\{\begin{array}{l}
\mathbf{u}(x, y, t)=\pi \sin (t+0.25)\left(\sin (2 \pi y) \sin (\pi x)^{2}, \sin (2 \pi x) \sin (\pi y)^{2}\right)^{T}, \\
p(x, y, t)=\sin (t+0.25) \cos (\pi x) \sin (\pi y) .
\end{array}\right.
$$

The computations are done with the P2/P1 Taylor-Hood element. In order to focus on the mesh change, the setting is as follows. The computations are started with different uniform meshes with mesh size $h_{0}$ (corresponding to 256, 1024 and 4096 elements, respectively) and uniform time step sizes $k$. The initial mesh is created by uniformly refining a given macro

\begin{tabular}{c|cccccc}
\hline$h$ & $\mathcal{E}_{N}^{\mathrm{ell}}$ & $\mathrm{EOC}$ & $\mathcal{E}_{N}^{\mathrm{T}}$ & $\mathrm{EOC}$ & $\mathcal{E}_{N}^{\mathrm{S}}$ & $\mathrm{EOC}$ \\
\hline 0.50000 & $3.9346 \mathrm{e}-01$ & - & $1.3879 \mathrm{e}-01$ & - & $1.6893 \mathrm{e}-01$ & \\
0.25000 & $1.0988 \mathrm{e}-01$ & 1.84 & $4.6089 \mathrm{e}-02$ & 1.59 & $5.1831 \mathrm{e}-02$ & 1.70 \\
0.12500 & $2.9590 \mathrm{e}-02$ & 1.89 & $1.1746 \mathrm{e}-02$ & 1.97 & $1.3949 \mathrm{e}-02$ & 1.89 \\
0.06250 & $7.6629 \mathrm{e}-03$ & 1.95 & $2.9427 \mathrm{e}-03$ & 2.00 & $3.6199 \mathrm{e}-03$ & 1.95 \\
0.03125 & $1.9437 \mathrm{e}-03$ & 1.98 & $7.3597 \mathrm{e}-04$ & 2.00 & $9.2003 \mathrm{e}-04$ & 1.98 \\
\hline
\end{tabular}

TABlE 2. Example 1: P1/P0 Crouzeix-Raviart elements combined with the backward Euler. The Stokes reconstruction estimator $\mathcal{E}_{N}^{\text {ell }}$, the time estimator $\mathcal{E}_{N}^{\mathrm{T}}$, the space estimator and $\mathcal{E}_{N}^{\mathrm{S}}$ and the corresponding EOCs. 


\begin{tabular}{c|cccc}
\hline$h$ & $\max _{n}\left\|\mathrm{e}^{n}\right\|$ & $\mathrm{EOC}$ & $\mathcal{E}_{N}$ & $\mathrm{EI}\left(t^{N}\right)$ \\
\hline 0.50000 & $9.6569 \mathrm{e}-02$ & - & $4.2795 \mathrm{e}+00$ & 44 \\
0.25000 & $2.8706 \mathrm{e}-02$ & 1.75 & $1.5760 \mathrm{e}+00$ & 55 \\
0.12500 & $7.8700 \mathrm{e}-03$ & 1.87 & $4.3589 \mathrm{e}-01$ & 55 \\
0.06250 & $2.0543 \mathrm{e}-03$ & 1.94 & $1.1240 \mathrm{e}-01$ & 55 \\
0.03125 & $5.3305 \mathrm{e}-04$ & 1.95 & $2.8550 \mathrm{e}-02$ & 54 \\
\hline
\end{tabular}

TABle 3. Example 2: P1/P0 Crouzeix-Raviart elements combined with the backward Euler. The $L^{\infty}\left(0,1 ; L^{2}(\Omega)\right)$-error for the velocity, the corresponding EOC, the total estimator $\mathcal{E}_{N}$ and the corresponding effectivity index $\operatorname{EI}\left(t^{N}\right)$.

\begin{tabular}{c|cccccc}
\hline$h$ & $\mathcal{E}_{N}^{\mathrm{ell}}$ & $\mathrm{EOC}$ & $\mathcal{E}_{N}^{\mathrm{T}}$ & $\mathrm{EOC}$ & $\mathcal{E}_{N}^{\mathrm{S}}$ & $\mathrm{EOC}$ \\
\hline 0.50000 & $2.6030 \mathrm{e}+00$ & - & $4.8472 \mathrm{e}-01$ & - & $1.1918 \mathrm{e}+00$ & \\
0.25000 & $7.6992 \mathrm{e}-01$ & 1.76 & $2.8652 \mathrm{e}-01$ & 0.76 & $5.1953 \mathrm{e}-01$ & 1.20 \\
0.12500 & $2.0209 \mathrm{e}-01$ & 1.93 & $8.4573 \mathrm{e}-02$ & 1.76 & $1.4922 \mathrm{e}-01$ & 1.80 \\
0.06250 & $5.1293 \mathrm{e}-02$ & 1.98 & $2.1992 \mathrm{e}-02$ & 1.94 & $3.9111 \mathrm{e}-02$ & 1.93 \\
0.03125 & $1.2940 \mathrm{e}-02$ & 1.99 & $5.6202 \mathrm{e}-03$ & 1.97 & $9.9896 \mathrm{e}-03$ & 1.97 \\
\hline
\end{tabular}

TABLE 4. Example 2: P1/P0 Crouzeix-Raviart elements combined with the backward Euler. The Stokes reconstruction estimator $\mathcal{E}_{N}^{\text {ell }}$, the time estimator $\mathcal{E}_{N}^{\mathrm{T}}$, the space estimator and $\mathcal{E}_{N}^{\mathrm{S}}$ and the corresponding EOCs.

\begin{tabular}{c|cccc}
\hline$h$ & $\max _{n}\left\|\mathrm{e}^{n}\right\|$ & $\mathrm{EOC}$ & $\mathcal{E}_{N}$ & $\mathrm{EI}\left(t^{N}\right)$ \\
\hline 0.50000 & $6.7276 \mathrm{e}-04$ & - & $1.7144 \mathrm{e}-01$ & 254 \\
0.25000 & $9.5060 \mathrm{e}-05$ & 2.83 & $2.5698 \mathrm{e}-02$ & 270 \\
0.12500 & $1.2202 \mathrm{e}-05$ & 2.96 & $3.2874 \mathrm{e}-03$ & 269 \\
0.06250 & $1.5383 \mathrm{e}-06$ & 2.99 & $9.2684 \mathrm{e}-03$ & 270 \\
\hline
\end{tabular}

TABLE 5. Example 2: P2/P1 Taylor-Hood elements combined with the backward Euler. The $L^{\infty}\left(0,1 ; L^{2}(\Omega)\right)$-error for the velocity, the corresponding EOC, the total estimator $\mathcal{E}_{N}$ and the corresponding effectivity index $\operatorname{EI}\left(t^{N}\right)$.

\begin{tabular}{c|cccccc}
\hline$h$ & $\mathcal{E}_{m}^{\mathrm{ell}}$ & EOC & $\mathcal{E}_{m}^{\mathrm{T}}$ & EOC & $\mathcal{E}_{m}^{\mathrm{S}}$ & EOC \\
\hline 0.50000 & $5.8452 \mathrm{e}-02$ & - & $8.7991 \mathrm{e}-02$ & - & $2.4994 \mathrm{e}-02$ & \\
0.25000 & $9.8056 \mathrm{e}-03$ & 2.58 & $1.1759 \mathrm{e}-02$ & 2.90 & $4.1331 \mathrm{e}-03$ & 2.60 \\
0.12500 & $1.2880 \mathrm{e}-03$ & 2.93 & $1.4720 \mathrm{e}-03$ & 3.00 & $5.2746 \mathrm{e}-04$ & 2.97 \\
0.06250 & $1.6365 \mathrm{e}-04$ & 2.97 & $1.8402 \mathrm{e}-04$ & 3.00 & $6.7154 \mathrm{e}-05$ & 2.97 \\
\hline
\end{tabular}

TABLE 6. Example 2: P2/P1 Taylor-Hood elements combined with the backward Euler. The Stokes reconstruction estimator $\mathcal{E}_{N}^{\text {ell }}$, the time estimator $\mathcal{E}_{N}^{\mathrm{T}}$, the space estimator and $\mathcal{E}_{N}^{\mathrm{S}}$ and the corresponding EOCs.

triangulation with the bisection method [4]. At the time instant $t_{\text {jump }}=1.28$ the mesh is derefined resulting in a coarsened mesh with mesh size $2 h_{0}$. This scenario is as in [12]. Since the pressure is the unknown most sensitive to mesh changes, we study the effect of mesh modification for this variable.

In Tables 7 and 8 , the $L^{2}\left(0, T ; L^{2}(\Omega)\right)$ and the coarsening estimator $\left(\sum_{n} k \gamma_{n}^{2}\right)^{1 / 2}$, respectively, are listed for various meshes and time step sizes $k$. Note that choosing $\Pi$ as the $L^{2}$ projection, 
orthogonality yields the relation

$$
\gamma_{n}^{2}:=\left\|k^{-1} U^{n-1}\right\|^{2}-\left\|k^{-1} \Pi^{n} U^{n-1}\right\|^{2},
$$

simplifying the computation of this term.

\begin{tabular}{l|rrrrr}
\hline$k$ & 0.0025 & 0.00125 & $6.25 \mathrm{e}-4$ & $3.125 \mathrm{e}-4$ & $1.5625 \mathrm{e}-04$ \\
\hline nt $=256$ & $2.2230 \mathrm{e}-02$ & $3.0141 \mathrm{e}-02$ & $4.1587 \mathrm{e}-02$ & $5.8022 \mathrm{e}-02$ & $8.1472 \mathrm{e}-02$ \\
$\mathrm{nt}=1024$ & $2.1866 \mathrm{e}-03$ & $2.5491 \mathrm{e}-03$ & $3.1449 \mathrm{e}-03$ & $4.0722 \mathrm{e}-03$ & $5.4670 \mathrm{e}-03$ \\
$\mathrm{nt}=4096$ & $4.8435 \mathrm{e}-04$ & $4.4688 \mathrm{e}-04$ & $4.5224 \mathrm{e}-04$ & $4.8128 \mathrm{e}-04$ & $5.3755 \mathrm{e}-04$
\end{tabular}

TABLE 7. Example $3: L^{2}\left(0, T ; L^{2}(\Omega)\right)$ pressure error for various meshes $(\mathrm{nt}=$ no. of elements) and time step sizes $k$.

From Tables 7 and 8 one infers that for small time step sizes (depending on the mesh size) the $L^{2}\left(L^{2}\right)$ error of the pressure as well as the coarsening estimator behave like $1 / \sqrt{k}$, thus showing the same asymptotic behavior with respect to the time step size. For larger time step sizes, the usual spatial and temporal discretization errors seem to dominate the pressure error compared to the error due to mesh change.

\begin{tabular}{l|rrrrr}
\hline$k$ & 0.0025 & 0.00125 & $6.25 \mathrm{e}-4$ & $3.125 \mathrm{e}-4$ & $1.5625 \mathrm{e}-04$ \\
\hline $\mathrm{nt}=256$ & 0.3878100 & 0.5484400 & 0.7756100 & 1.0969000 & 1.5512000 \\
$\mathrm{nt}=1024$ & 0.0584790 & 0.0827020 & 0.1169600 & 0.1654100 & 0.2339200 \\
$\mathrm{nt}=4096$ & 0.0079339 & 0.0112200 & 0.0158680 & 0.0224410 & 0.0317370 \\
\hline
\end{tabular}

TABLE 8. Example 3: coarsening estimator $\left(\sum_{n} k \gamma_{n}^{2}\right)^{1 / 2}$ for various meshes (nt $=$ no. of elements) and time step sizes $k$.

To get more insight, let us have a closer look at the temporal evolution of the error and the estimator. In [12] it was shown that a de-refinement of the mesh results in a jump of the pressure error of the order $1 / k$. This behavior is indeed confirmed by our experiments. In Fig. 2 the $L^{2}(\Omega)$-errror of the pressure is shown as a function of time. The huge jump in the error at $t_{\text {jump }}$ is clearly visible.

More quantitative information is given in Tables 9 and 10. In Table 9 the jumps $\delta e_{p}$ in the pressure error are listed for various $k$ and $h_{0}$. Here, $\delta e_{p}$ is defined as the "extra" contribution to the pressure error by the mesh change:

$$
\delta e_{p}:=\left\|P^{n_{0}}-p\left(t_{\text {jump }}\right)\right\|-\left\|P^{n_{0}-1}-p\left(t_{\text {jump }}-k\right)\right\|,
$$

where $n_{0}$ corresponds to the time instant $t_{\text {jump }}=n_{0} k$. Notice that our theory does not provide error control for $\delta e_{p}$. This term is used in our experiments only as tool to quantify the observed jump in the pressure. The comparison then to our coarsening estimator is indicative only in order to access whether our estimator can detect or not the jump in pressure.

In Table 10, the coarsening estimator terms $\gamma_{n_{0}}:=\left\|k^{-1}\left(\Pi^{n_{0}}-I\right) U^{n_{0}-1}\right\|$ are reported. Notice that in this example $\gamma_{n}=0$ for $n \neq n_{0}$.

From Table 9 one deduces the following behavior of $\delta e_{p}$. If the mesh is fine enough, the "extra contribution" of the error is negligibly small. However, from a certain small time step size $k$ (depending on $h_{0}$ ), the extra contribution to the error behaves like $1 / k$ (reading the rows of Table 9$)$. In contrast, fixing a time step size and reading the columns, $\delta e_{p}$ is decreasing. 


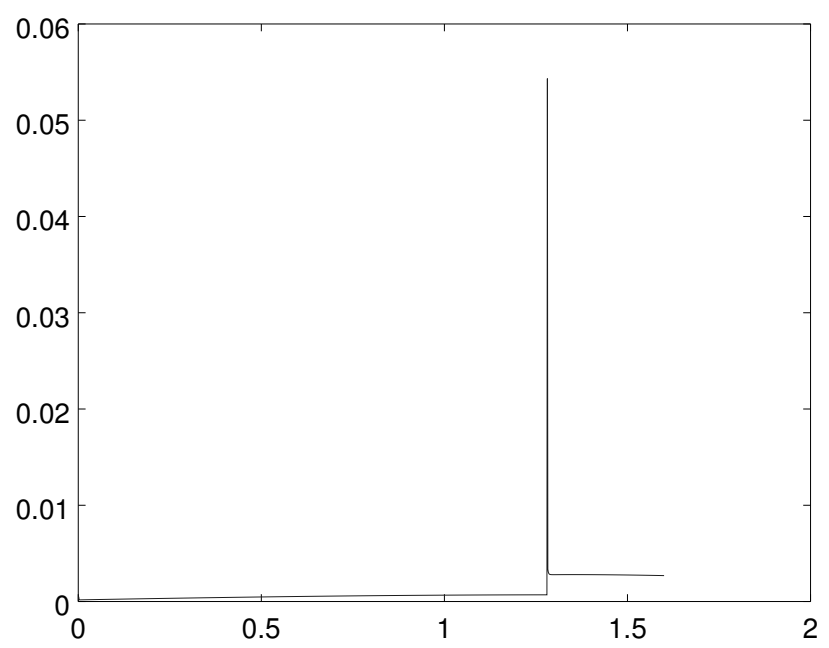

FiguRE 2. Example 3: $L^{2}(\Omega)$ pressure error versus time (initial mesh with 1024 elements, $k=1.25 \mathrm{e}-3$, P2/P1 Taylor-Hood element).

\begin{tabular}{l|rrrrr}
\hline$k$ & $2 . \mathrm{e}-2$ & $1 . \mathrm{e}-2$ & $5 . \mathrm{e}-3$ & $2.5 \mathrm{e}-3$ & $1.25 \mathrm{e}-3$ \\
\hline $\mathrm{nt}=256$ & $5.2902 \mathrm{e}-02$ & $1.0772 \mathrm{e}-01$ & $2.1290 \mathrm{e}-01$ & $4.1863 \mathrm{e}-01$ & $8.2561 \mathrm{e}-01$ \\
nt $=1024$ & $3.5000 \mathrm{e}-06$ & $5.8377 \mathrm{e}-03$ & $1.3334 \mathrm{e}-02$ & $2.7093 \mathrm{e}-02$ & $5.3634 \mathrm{e}-02$ \\
nt= 4096 & $5.0000 \mathrm{e}-07$ & $2.0000 \mathrm{e}-07$ & $3.4452 \mathrm{e}-04$ & $1.5053 \mathrm{e}-03$ & $3.4365 \mathrm{e}-03$ \\
\hline
\end{tabular}

TABLE 9. Example 3: "extra" pressure error $\delta e_{p}$ (6.5) for various meshes $(\mathrm{nt}=$ no. of elements) and time step sizes $k$.

A similar behavior can be observed for the coarsening estimator $\gamma$. However, the $1 / k$ increase is valid for all $h_{0}$. For fixed time step size, $\gamma$ decreases approximately like $h_{0}^{3}$, which is the order one would expect for the P2/P1 Taylor-Hood element.

We conclude that, in this experiment, our estimator has the right qualitative behavior and captures the significant increase of the error introduced by the mesh change.

\begin{tabular}{l|rrrrr}
\hline$k$ & $2 . \mathrm{e}-2$ & $1 . \mathrm{e}-2$ & $5 . \mathrm{e}-3$ & $2.5 \mathrm{e}-3$ & $1.25 \mathrm{e}-3$ \\
\hline $\mathrm{nt}=256$ & 0.969690 & 1.939200 & 3.878200 & 7.756300 & 15.512000 \\
$\mathrm{nt}=1024$ & 0.146180 & 0.292380 & 0.584780 & 1.169600 & 2.339200 \\
$\mathrm{nt}=4096$ & 0.019830 & 0.039666 & 0.079336 & 0.158680 & 0.317360
\end{tabular}

TABLE 10. Example 3: mesh change estimator $\gamma$ for various meshes $(\mathrm{nt}=$ no. of elements) and time step sizes $k$.

\section{REFERENCES}

[1] G. Akrivis, C. Makridakis, and R. H. Nochetto. A posteriori error estimates for the Crank-Nicolson method for parabolic equations. Math. Comp., 75:511-531, 2006.

[2] G. Akrivis, C. Makridakis, and R. H. Nochetto. Optimal order a posteriori error estimates for a class of Runge-Kutta and Galerkin methods. Numer. Math., 114:133 - 160, 2009. 
[3] M. S. Alnaes, J. Blechta, J. Hake, A. Johansson, B. Kehlet, A. Logg, C. Richardson, J. Ring, M. Rognes, and G. N. Wells. The fenics project version 1.5. Archive of Numerical Software, 3(100), 2015.

[4] E. Bänsch. Local mesh refinement in 2 and 3 dimensions. Impact Comput. Sci. Engrg., 3(3):181-191, 1991.

[5] E. Bänsch. Simulation of instationary, incompressible flows. In Proceedings of the Algoritmy'97 Conference on Scientific Computing (Zuberec), volume 67, pages 101-114, 1998.

[6] E. Bänsch, F. Karakatsani, and C. Makridakis. A posteriori error control for fully discrete Crank-Nicolson schemes. SIAM J. Numer. Anal., 50(6):2845-2872, 2012.

[7] E. Bänsch, F. Karakatsani, and C. Makridakis. The effect of mesh modification in time on the error control of fully discrete approximations for parabolic equations. Appl. Numer. Math., 67:35-63, 2013.

[8] E. Bänsch, P. Morin, and R. H. Nochetto. An adaptive Uzawa FEM for the Stokes problem: convergence without the inf-sup condition. SIAM J. Numer. Anal., 40(4):1207-1229, 2002.

[9] E. Bänsch and A. Schmidt. Simulation of dendritic crystal growth with thermal convection. Interfaces Free Bound., 2(1):95-115, 2000.

[10] C. Bernardi and T. Sayah. A posteriori error analysis of the time-dependent Stokes equations with mixed boundary conditions. IMA J. Numer. Anal., 35(1):179-198, 2015.

[11] C. Bernardi and R. Verfürth. A posteriori error analysis of the fully discretized time-dependent Stokes equations. M2AN Math. Model. Numer. Anal., 38(3):437-455, 2004.

[12] M. Besier and W. Wollner. On the pressure approximation in nonstationary incompressible flow simulations on dynamically varying spatial meshes. Int. J. Numer. Meth. Fluids, 2011.

[13] A. Brenner, E. Bänsch, and M. Bause. A priori error analysis for finite element approximations of the Stokes problem on dynamic meshes. IMA J. Numer. Anal., 34(1):123-146, 2014.

[14] S. C. Brenner and L. R. Scott. The mathematical theory of finite element methods. Springer-Verlag, New York, 1994.

[15] P. Chatzipantelidis, C. Makridakis, and M. Plexousakis. A-posteriori error estimates for a finite volume method for the Stokes problem in two dimensions. Appl. Numer. Math., 46(1):45-58, 2003.

[16] M. Crouzeix and P.-A. Raviart. Conforming and nonconforming finite element methods for solving the stationary Stokes equations. I. Rev. Française Automat. Informat. Recherche Opérationnelle Sér. Rouge, 7(R3):33-75, 1973 .

[17] E. Dari, R. Durán, and C. Padra. Error estimators for nonconforming finite element approximations of the Stokes problem. Math. Comp., 64(211):1017-1033, 1995.

[18] R. Dautray and J.-L. Lions. Mathematical analysis and numerical methods for science and technology. Vol. 6. Springer-Verlag, Berlin, 1993. Evolution problems. II, With the collaboration of Claude Bardos, Michel Cessenat, Alain Kavenoky, Patrick Lascaux, Bertrand Mercier, Olivier Pironneau, Bruno Scheurer and Rémi Sentis.

[19] T. Dupont. Mesh modification for evolution equations. Mathematics of Computation, 39:85-107, 1982.

[20] V. Girault and P.-A. Raviart. Finite element methods for Navier-Stokes equations. Theory and algorithms, volume 5 of Springer Series in Computational Mathematics. Springer-Verlag, Berlin, 1986.

[21] A. Hannukainen, R. Stenberg, and M. Vohralík. A unified framework for a posteriori error estimation for the Stokes problem. Numer. Math., 122(4):725-769, 2012.

[22] J. G. Heywood and R. Rannacher. Finite element approximation of the nonstationary Navier-Stokes problem. I. Regularity of solutions and second-order error estimates for spatial discretization. SIAM J. Numer. Anal., 19(2):275-311, 1982.

[23] J. G. Heywood and R. Rannacher. An analysis of stability concepts for the Navier-Stokes equations. J. Reine Angew. Math., 372:1-33, 1986.

[24] V. John. A posteriori $L^{2}$-error estimates for the nonconforming $P_{1} / P_{0}$-finite element discretization of the Stokes equations. J. Comput. Appl. Math., 96(2):99-116, 1998.

[25] O. Karakashian and C. Makridakis. A space-time finite element method for the nonlinear Schrödinger equation: the discontinuous Galerkin method. Math. Comp., 67(222):479-499, 1998.

[26] F. Karakatsani. A posteriori error estimates for the fractional-step $\vartheta$-scheme for linear parabolic equations. IMA J. Numer. Anal., 32(1):141-162, 2012.

[27] F. Karakatsani and C. Makridakis. A posteriori estimates for approximations of time-dependent Stokes equations. IMA Journal of Numerical Analysis, 27(4):741-764, 2007.

[28] O. Lakkis and C. Makridakis. Elliptic reconstruction and a posteriori error estimates for fully discrete linear parabolic problems. Math. Comp., 75(256):1627-1658, 2006.

[29] C. Makridakis and R. H. Nochetto. Elliptic reconstruction and a posteriori error estimates for parabolic problems. SIAM J. Numer. Anal., 41(4):1585-1594 (electronic), 2003. 
[30] A. Schmidt and K. G. Siebert. Design of adaptive finite element software, volume 42 of Lecture Notes in Computational Science and Engineering. Springer-Verlag, Berlin, 2005. The finite element toolbox ALBERTA, With 1 CD-ROM (Unix/Linux).

[31] C. Taylor and P. Hood. A numerical solution of the Navier-Stokes equations using the finite element technique. Internat. J. Comput. \& Fluids, 1(1):73-100, 1973.

[32] R. Temam. Navier-Stokes equations. Theory and numerical analysis. AMS Chelsea Publishing, Providence, RI, 2001. Reprint of the 1984 edition.

[33] R. Verfürth. A posteriori error estimators for the Stokes equations. Numer. Math., 55(3):309-325, 1989.

[34] R. Verfürth. A posteriori error analysis of space-time finite element discretizations of the time-dependent Stokes equations. Calcolo, 47(3):149-167, 2010.

[35] R. Verfürth. A posteriori error estimation techniques for finite element methods. Numerical Mathematics and Scientific Computation. Oxford University Press, Oxford, 2013.

Applied Mathematics III Cauerstr. 11 D-91058 Erlangen Germany

E-mail address: baensch@math.fau.de

Department of Mathematics, Faculty of Science \& Engineering, University of Chester Thornton Science Park, Pool Lane, Ince Chester CH2 4NU

E-mail address: f.karakatsani@chester.ac.uk

School for Mathematical and Physical Sciences, University of Sussex, Brighton, BN1 9QH, UK E-mail address: c.makridakis@sussex.ac.uk 